DCPT- $02 / 31$

FFUOV-02/05

1 August 2002

\title{
On the Dielectric Effect for Gravitational Waves
}

\author{
Bert Janssen $^{a \text {, 円 and Yolanda Lozano }}{ }^{b, \text {, }}$ \\ ${ }^{a}$ Department of Mathematical Sciences, \\ South Road, Durham DH1 3LE, United Kingdom \\ ${ }^{b}$ Departamento de Física, Universidad de Oviedo \\ Avda. Calvo Sotelo 18, 33007 Oviedo, Spain
}

\begin{abstract}
We argue that the dielectric effect, mostly studied for systems of coincident D-branes, is also extendible to configurations of multiple gravitational waves. We provide some evidence that Matrix string theory has an alternative interpretation as describing also, in the static gauge, multiple Type IIA gravitational waves. Starting with the linearised action of Matrix string theory in a weakly curved background, we identify the non-Abelian couplings of multiple coinciding gravitons to weak background fields, both in Type IIA and in Type IIB, and we use them to study various dielectric configurations from the point of view of the expanding gravitons. We also identify, in the Abelian limit, some non-perturbative correction terms to the Abelian gravitational wave action.
\end{abstract}

\footnotetext{
${ }^{1}$ E-mail address: bert.janssen@durham.ac.uk

${ }^{2}$ E-mail address: yolanda@string1.ciencias.uniovi.es
} 


\section{Introduction}

The dielectric effect, i.e. the idea that a collection of branes can undergo an expansion into a higher-dimensional brane under the influence of an external flux tube, has recently attracted a lot of attention. This effect was first explored in [1], where Emparan constructed solutions of the Abelian Born-Infeld theory, describing a single $\mathrm{D} p$-brane with $N$ units of Born-Infeld flux dissolved in its world volume, that could be interpreted in terms of expanded fundamental strings or expanded $\mathrm{D}(p-2)$-branes. These solutions carry no global charge with respect to the background $\mathrm{R}-\mathrm{R}(p+1)$-form potential that needs to be switched on in order to prevent the $\mathrm{D} p$-brane from collapsing, but do have a non-zero local charge, which results in a non-zero dipole moment, that renders the configuration stable.

A microscopic description of this effect, from the point of view of the lower-dimensional $\mathrm{D}(p-2)$-branes, was provided some years later by Myers [2]. He observed that a system of coincident D-branes can develop multipole moment couplings to R-R fields that would normally be associated to higher-dimensional branes. From this point of view, the expansion is due to the fact that the transverse coordinates to the coincident D-branes are matrix-valued, which allows the D-branes to couple to background R-R fields $C^{(q-1)}$, with $q>p(p, q$ even (odd) in IIA (IIB)) [2, 3]. Myers constructed a solution of the non-Abelian world volume effective action, describing $N$ coinciding $\mathrm{D}(p-2)$-branes under the influence of an external R-R $(p+2)$-form field strength. He interpreted this non-Abelian solution as the expansion of the $N \mathrm{D}(p-2)$ branes into a $\mathrm{D} p$-brane of topology $\mathbb{R}^{1, p-2} \times S_{N C}^{2}$, where $S_{N C}^{2}$ denotes the noncommutative, or fuzzy 2-sphere. The $\mathrm{D} p$-brane is, as in the Abelian description, uncharged with respect to the $\mathrm{R}-\mathrm{R}(p+1)$-form potential, but has a non-zero dipole moment. Hence the name of dielectric $\mathrm{D} p$-brane, introduced by Myers. Other expanded brane configurations, involving more general fuzzy cosets, have been considered in 迎]

Both descriptions of the dielectric effect, Abelian and non-Abelian, have complementary ranges of validity [2], that coincide in the limit where the number $N$ of $\mathrm{D}(p-2)$-branes goes to infinity, in which the non-commutative nature of the fuzzy two-sphere is lost.

Supergravity solutions corresponding to expanding branes have been constructed, specifically for D4-branes [5, 6] and fundamental strings [6] expanding into a dielectric D6-brane of topology $\mathbb{R}^{1,4} \times S^{2}$ and $\mathbb{R}^{1,1} \times S^{5}$ respectively. Again here, the physical properties of these supergravity solutions, such as their stability and the size of the fuzzy spheres, match with the Abelian world volume analysis of the dielectric D6-brane [5, 7].

A microscopical description of dielectric configurations involving fundamental strings has also been proposed. Expanding fundamental strings have been studied at the non-Abelian, microscopic level in [8, 9] (see also [10]), using Matrix string theory in a weakly curved background. Matrix string theory [11] turns out to give the right description of fundamental strings in terms of matrix-valued coordinates, and the linear couplings to weak background fields generate precisely the non-Abelian couplings responsible for the dielectric effect. Dielectric solutions of fundamental strings expanding into D3-branes and D4-branes were constructed in [9].

We also argued in [9], and we will provide further evidence in this paper, that Matrix string theory, in the static gauge, describes coinciding Type IIA gravitational waves. Indeed, we will see that Matrix string theory has an alternative interpretation as describing also the dynamics of multiple gravitational waves. Since Matrix string theory describes string states with fixed light cone momentum, it is not surprising that, in some limit, it can effectively describe gravitational waves. 
A possible dielectric effect for gravitational waves seems to be related to another topic that has recently received a lot of attention: giant gravitons [12]. One can roughly think of a giant graviton configuration in terms of gravitons expanded into a $p$-brane with topology $\mathbb{R} \times S^{p}$, carrying angular momentum and dipole moment. This configuration breaks supersymmetry in the same way as a point-like graviton propagating with the same velocity, but in $A d S_{m} \times$ $S^{p+2}$ spacetimes, where the $p$-brane expands into the $S^{p+2}$ part of the geometry, it has associated a maximum angular momentum. As is well-known, these configurations were in fact proposed in [12] as a way to satisfy the stringy exclusion principle implied by the AdS/CFT correspondence.

The giant graviton analysis of [12] was in terms of the Abelian gauge theory relevant to the description of the expanded brane. Since massless particles, in particular gravitons, are the source terms for gravitational waves, it seems natural to assume that the dielectric effect for gravitational waves provides a microscopic picture for giant gravitons. Such a microscopical description has so far only been given in some particular case. In [13] a solution corresponding to $N$ M-theory gravitons expanding into an M2-brane of topology $\mathbb{R}^{1,1} \times S_{N C}^{2}$ was studied in terms of D0-branes expanding into a D2-brane, and then uplifted to M-theory. This microscopical effect was referred to as a magnetic moment effect, because the expanded brane carries a magnetic, instead of an electric, dipole moment.

A second type of giant gravitons, in which the expanded brane has an electric dipole moment, was considered in [14, 15]. In $A d S_{m} \times S^{n}$ spacetimes, these gravitons expand the $A d S$ part of the geometry, and therefore do not provide a realisation of the stringy exclusion principle. They are referred to in the literature as dual giant gravitons. Other giant graviton solutions in various backgrounds have been studied elsewhere in the literature [16, 17, 18].

In this paper, we aim at describing dielectric configurations from the point of view of expanding gravitational waves. We will argue that it is possible to describe multiple gravitational waves using Matrix theory. As we will discuss, Matrix string theory describes, in the static gauge, coinciding Type IIA gravitational waves. Therefore, taking the linearised action of Type IIA Matrix string theory in a weakly curved background, constructed in [8] and [9], we can identify the extra non-perturbative couplings of non-Abelian gravitons to background fields, and use them to study dielectric configurations. This same analysis can be done in Type IIB using T-duality. In this paper we have constructed the linearised world volume couplings of multiple gravitons to closed string fields in the two Type II theories. We have to stress, however, that it would be necessary to go beyond the linear order calculation presented in this paper in order to study the giant graviton configurations of [12]-15], given that our couplings represent a linear perturbation to the Minkowski spacetime, and are therefore not suitable to describe $A d S_{m} \times S^{n}$ backgrounds.

We start in section 2 by reviewing in some detail the T-duality relation between fundamental strings and gravitational waves in the Abelian case, since this will be of use later. We show here that the effective action of a gravitational wave can be defined in terms of a gauged sigma model in which the direction of propagation of the wave enters as a special Killing direction. This action is related through a Legendre transformation to the usual action for the ten-dimensional massless particle. In section 3, we present the derivation of the effective action for Type IIA multiple gravitational waves from the Matrix string theory action. As a check we show that at weak coupling this action reproduces the linearised, Abelian effective action derived in section 2. Some of the couplings in this action were also derived by one of us [19], using various string dualities. In section 4, a further T-duality takes us to the Type IIB theory, where we construct the Matrix theory action that describes multiple Type 
IIB gravitational waves. This action is S-duality invariant, as predicted by the analysis of the Type IIB supersymmetry algebra, and reduces also, at weak coupling, to a certain linearised Abelian effective action for a massless particle, derived in section 2. In section 5, we consider various dielectric solutions that arise from the non-Abelian couplings derived in this paper. We also comment on the duality relations between these solutions, as well as on their microscopical picture interpretation for giant gravitons.

\section{T-duality between fundamental strings and gravitational waves}

We start in this section discussing in some detail the construction of the effective action that describes a single gravitational wave. It is well known that gravitational waves and fundamental strings are related by a T-duality transformation along the direction of propagation of the wave. This works simply at the level of the supergravity solutions [20]. The supergravity solution corresponding to a gravitational wave propagating in the $x$ direction,

$$
d s^{2}=-(2-H) d t^{2}+H d x^{2}-2(1-H) d t d x+d y_{1}^{2}+\ldots+d y_{8}^{2},
$$

is mapped under T-duality along this direction into the supergravity solution of a fundamental string,

$$
\begin{aligned}
& d s^{2}=H^{-1}\left(-d t^{2}+d x^{2}\right)+d y_{1}^{2}+\ldots+d y_{8}^{2}, \\
& e^{-2 \phi}=H, \quad B_{0 x}=H^{-1}-1,
\end{aligned}
$$

where $x$ is now the spatial world volume direction of the string.

At the level of the effective actions, the procedure is a little more subtle. It is well known that a T-duality transformation applied on the non-linear sigma model describing a string propagating in a non-trivial background yields a new non-linear sigma model describing a string moving in the dual background defined by Buscher's rules. Instead of performing the most general analysis, however, it is possible to start with a very specific string state, a state with zero momentum and non-zero winding number, and apply a T-duality transformation. The string state thus obtained is a state with only momentum, which can be described effectively by a gravitational wave type of action.

Using this duality connection between string states with winding number and string states with momentum, one can simply construct the effective action associated to the wave from the

Nambu-Goto action for a fundamental string, keeping in mind that the action thus derived is only an effective action for a specific string state. As we show next, one is led in this manner to a gauged sigma model, in which the direction of propagation of the wave appears as a special isometric direction, in such a way that the gravitational field is transversal to the direction of propagation.

The Nambu-Goto action of the string is given in our notation by:

$$
S_{F 1}=-T_{1} \int d^{2} \sigma \sqrt{\left|\operatorname{det}\left(\partial_{\alpha} X^{\mu} \partial_{\beta} X^{\nu} g_{\mu \nu}\right)\right|}+\frac{1}{2} T_{1} \int d^{2} \sigma \varepsilon^{\alpha \beta} \partial_{\alpha} X^{\mu} \partial_{\beta} X^{\nu} B_{\mu \nu} .
$$

If we assume that the ninth direction is compact and that the string is wound $m$ times around this direction, we can make a split in the target space coordinates,

$$
X^{\mu}(\tau, \sigma)=\left(X^{a}(\tau), X^{9}(\sigma)\right)=\left(X^{a}(\tau), m \sigma\right),
$$


and T-dualise along $X^{9}$. Using Buscher's rules in the kinetic term of the action we arrive at:

$$
\begin{aligned}
& \operatorname{det}\left(\partial_{\alpha} X^{\mu} \partial_{\beta} X^{\nu} g_{\mu \nu}\right)=\partial_{\tau} X^{a} \partial_{\tau} X^{b}\left(g_{99} g_{a b}-g_{a 9} g_{b 9}\right) \\
& \longrightarrow \partial_{\tau} X^{\mu} \partial_{\tau} X^{\nu} \frac{1}{g_{99}}\left(g_{\mu \nu}-\frac{g_{\mu 9} g_{\nu 9}}{g_{99}}\right) \equiv \partial X^{\mu} \partial X^{\nu} \mathcal{G}_{\mu \nu} .
\end{aligned}
$$

Here $\partial$ denotes derivation with respect to $\tau$ and $\mathcal{G}_{\mu \nu}$ is a reduced metric, which we can write as

$$
\mathcal{G}_{\mu \nu}=g_{\mu \nu}-k^{-2} k_{\mu} k_{\nu}
$$

introducing a Killing vector pointing along the $X^{9}$, or $x$, direction: $k^{\mu}=\delta_{x}^{\mu}$, so that we have

$$
k_{\mu}=g_{\mu x}, \quad k^{2}=k_{x}=g_{x x} .
$$

Similarly, the Wess-Zumino term gives:

$$
B_{a x} \rightarrow-\frac{g_{a x}}{g_{x x}}=-\frac{k_{a}}{k^{2}}
$$

We then arrive at:

$$
S_{W}=-m T_{0} \int d \tau\left\{k^{-1} \sqrt{\left|\partial X^{\mu} \partial X^{\nu} \mathcal{G}_{\mu \nu}\right|}+k^{-2} k_{a} \partial X^{a}\right\} .
$$

It is straightforward to see that the components of $\mathcal{G}_{\mu \nu}$ in the $x$ direction vanish,

$$
k^{\mu} \mathcal{G}_{\mu \nu}=0
$$

which states that the gravitational field is transversal to the direction of propagation of the wave, since we are going to identify soon the $x$ direction with the direction of propagation. The reduced metric projects out the world volume scalar $X^{9}$, thus removing the world volume degree of freedom corresponding to the direction of propagation of the wave. The action (2.9) can in fact be written as a gauged sigma model, invariant under local transformations along the $x$ direction:

$$
S_{W}=-m T_{0} \int d \tau\left\{k^{-1} \sqrt{\left|\mathcal{D} X^{\mu} \mathcal{D} X^{\nu} g_{\mu \nu}\right|}+A-\partial X^{9}\right\}
$$

$\mathcal{D} X^{\mu}$ denote gauge covariant derivatives

$$
\mathcal{D} X^{\mu} \equiv \partial X^{\mu}-A k^{\mu} \equiv \partial X^{\mu}-k^{-2} k_{\rho} \partial X^{\rho} k^{\mu},
$$

with respect to the scaling symmetry

$$
\delta X^{\mu}=\Lambda(\tau) k^{\mu}, \quad \delta A=\partial \Lambda(\tau) .
$$

Note that the covariant derivatives reduce to ordinary derivatives for $\mu \neq x$, while for $\mu=x$ we have $\mathcal{D} X^{9}=-k^{-2} k_{a} \partial X^{a}$. The action (2.11) is manifestly invariant under the transformations (2.13). Gauged sigma models of the type of (2.9) and (2.11) have been used before in order to describe the effective actions of Kaluza-Klein monopoles 21] and M-branes in massive eleven-dimensional supergravity [22, 23]. 
The string winding number, $m$, is now the momentum number of the wave in the isometry direction, given that

$$
m=\frac{1}{T_{0}} \frac{\delta S_{W}}{\delta\left(\partial X^{9}\right)}
$$

a fact that identifies the isometry direction with the direction of propagation of the wave.

The action (2.11) can in fact be rewritten as the action of the massless ten-dimensional particle. In order to see this, we should think of (2.11) as a Legendre transformed action in which the dependence on $\partial X^{9}$ has been replaced by a dependence on its conjugate momentum $P_{9}$, but in such a way that a total derivative term $P_{9} \partial X^{9}$ is kept in order to have a gauge invariant Lagrangian [22]. We then write (2.11) as:

$$
S_{W}\left[P_{9}\right]=-\int d \tau P_{9}\left\{k^{-1} \sqrt{\left|\mathcal{D} X^{\mu} \mathcal{D} X^{\nu} g_{\mu \nu}\right|}+A-\partial X^{9}\right\}
$$

Integration over $X^{9}$ fixes $P_{9}$ to a constant. It is convenient now to introduce an auxiliary metric $\gamma$ on the world line and define an action $S_{W}\left[P_{9}, \gamma\right]$ such that 2.15) is recovered upon integration on $\gamma$ :

$$
\begin{gathered}
S_{W}\left[P_{9}, \gamma\right]=-\frac{m T_{0}}{2} \int d \tau \\
\sqrt{|\gamma|}\left\{\gamma^{-1} \mathcal{D} X^{\mu} \mathcal{D} X^{\nu} g_{\mu \nu}+k^{-2}\left(\frac{P_{9}}{m T_{0}}\right)^{2}\right\} \\
+\int d \tau P_{9}\left(\partial X^{9}-A\right) .
\end{gathered}
$$

From this action we easily restore the dependence on $\partial X^{9}$ through a Legendre transformation:

$$
S[\gamma]=\int d \tau\left\{-P_{9} \partial X^{9}+L\left[P_{9}, \gamma\right]\right\}, \quad \partial X^{9}=\frac{\delta L\left[P_{9}, \gamma\right]}{\delta P_{9}},
$$

in such a way that, after integrating $P_{9}$, we obtain the action for the massless ten-dimensional particle:

$$
S[\gamma]=-\frac{m T_{0}}{2} \int d \tau \sqrt{|\gamma|} \gamma^{-1} \partial X^{\mu} \partial X^{\nu} g_{\mu \nu}
$$

We have thus checked that the action (2.11), T-dual to the Nambu-Goto action (2.3), describes a massless particle in the ten-dimensional spacetime. When the direction of propagation and the momentum carried by the particle are specified, the more suitable description is in terms of the action 2.11), in which the direction of propagation is singled out as a special isometric direction, and the momentum arises as the charge with respect to the background field $k_{\mu} / k^{2}$.

We now compute for future reference the expansion of the effective action (2.11) up to linear order in the background fields. For small fluctuations in the background metric $g_{\mu \nu} \approx$ $\eta_{\mu \nu}+h_{\mu \nu}$, we find

$$
\begin{aligned}
S_{W}^{\operatorname{lin}}= & -m T_{0} \int d \tau\left\{1-\frac{1}{2} \dot{X}^{2}-\frac{1}{2} h_{00}-\frac{1}{4} h_{00} \dot{X}^{2}-h_{0 i} \dot{X}^{i}-\frac{1}{2} h_{i j} \dot{X}^{i} \dot{X}^{j}\right. \\
& \left.-\frac{1}{2} h_{x x}+\frac{1}{4} h_{x x} \dot{X}^{2}+h_{0 x}+h_{i x} \dot{X}^{i}+\ldots\right\}
\end{aligned}
$$

where the indices $i, j$ run from $1, \ldots, 8 \dot{X}^{2} \equiv \eta_{i j} \dot{X}^{i} \dot{X}^{j}$ and the dot indicates derivation with respect to the time-like coordinate $\tau$. 


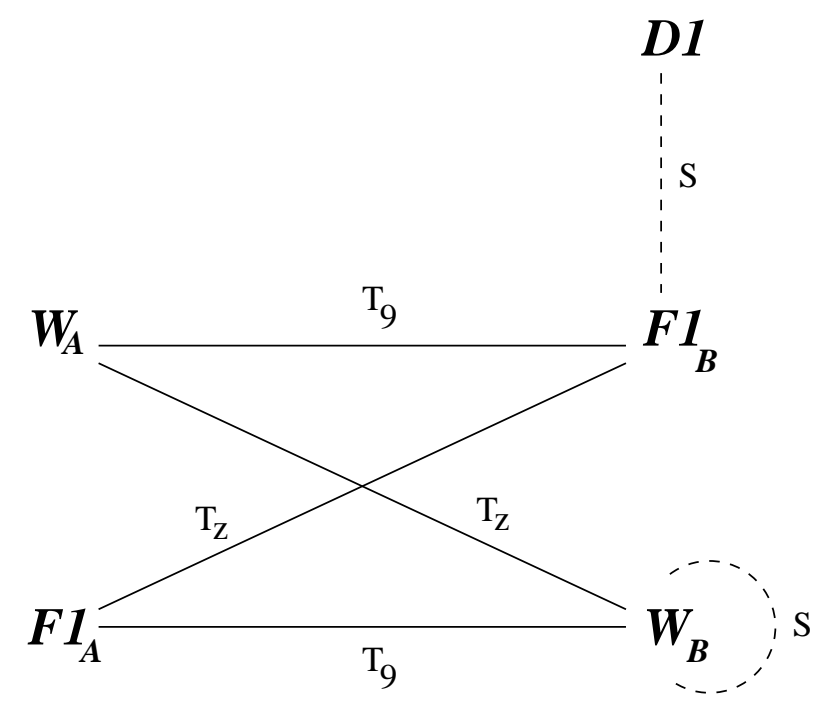

Figure 1: The T-duality relations between fundamental strings and gravitational waves in the Type II theories. $T_{9}$ denotes T-duality along $X^{9}$, the world volume direction of the string or propagation direction of the wave, while $T_{z}$ denotes T-duality along a direction transverse to the string or the wave. Also shown is the S-duality relation between the D-string and the fundamental string of Type IIB and the S-duality invariance of the gravitational wave in Type IIB.

Let us now apply a T-duality transformation in a direction transverse to the direction of propagation of the wave that we have just studied. From (2.1) we easily see that the super-

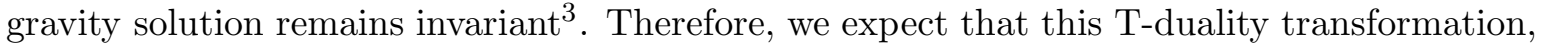
when applied to the action (2.11), will yield again the action for a gravitational wave. In fact, the T-duality transformation gives rise to a complicated action containing two gauged isometries, but which can again be related, through a Legendre transformation, to the (dimensional reduction of the) action for the massless ten-dimensional particle. We present this calculation in detail. Indeed, we will see that the non-Abelian extension of this action, with two isometries, arises as the Matrix theory description of multiple Type IIB waves that we will present in section 4.

Applying T-duality on the action (2.11) along a transverse direction $Z$ we find:

$$
\begin{aligned}
S_{W}= & -m T_{0} \int d \tau\left\{|l|\left|k^{2} l^{2}-(k . l)^{2}+\left(\mathrm{i}_{k} \mathrm{i}_{l} B\right)^{2}\right|^{-1 / 2} .\right. \\
& \cdot \sqrt{\left|\mathcal{D} X^{\mu} \mathcal{D} X^{\nu} g_{\mu \nu}+\frac{k^{2}-(k . l)^{2} / l^{2}}{k^{2} l^{2}-(k . l)^{2}+\left(\mathrm{i}_{k} \mathrm{i}_{l} B\right)^{2}} \tilde{\mathcal{F}}^{2}\right|} \\
& \left.+A^{(1)}-\partial X^{9}+\frac{\mathrm{i}_{k} \mathrm{i}_{l} B}{k^{2} l^{2}-(k . l)^{2}+\left(\mathrm{i}_{k} \mathrm{i}_{l} B\right)^{2}} \tilde{\mathcal{F}}\right\} .
\end{aligned}
$$

This action contains two isometric directions, the direction of propagation of the original wave, $X^{9}$, and the transverse direction $Z$ in which we performed the T-duality. We have denoted the corresponding Killing vectors as $k^{\mu}=\delta_{x}^{\mu}$ as before and $l^{\mu}=\delta_{z}^{\mu}$. The gauge

\footnotetext{
${ }^{3}$ An overview of the T-duality relations between gravitational waves and fundamental strings can be found in Figure 1.
} 
covariant derivatives $\mathcal{D} X^{\mu}$ are now defined in such a way that they gauge the two local isometric transformations generated by $k^{\mu}$ and $l^{\mu}$

$$
\delta X^{\mu}=\Lambda^{(1)}(\tau) k^{\mu}+\Lambda^{(2)}(\tau) l^{\mu},
$$

that is,

$$
\begin{aligned}
\mathcal{D} X^{\mu} & \equiv \partial X^{\mu}-A^{(1)} k^{\mu}-A^{(2)} l^{\mu} \\
& \equiv \partial X^{\mu}-\frac{l^{2}\left(k_{\rho} \partial X^{\rho}\right)-(k \cdot l)\left(l_{\rho} \partial X^{\rho}\right)}{k^{2} l^{2}-(k \cdot l)^{2}} k^{\mu}-\frac{k^{2}\left(l_{\rho} \partial X^{\rho}\right)-(k \cdot l)\left(k_{\rho} \partial X^{\rho}\right)}{k^{2} l^{2}-(k \cdot l)^{2}} l^{\mu} .
\end{aligned}
$$

$\mathcal{D} X^{\mu} \mathcal{D} X^{\nu} g_{\mu \nu}$ can equivalently be written in terms of a reduced metric, $\partial X^{\mu} \partial X^{\nu} \mathcal{G}_{\mu \nu}$, such that its components in the $x$ and $z$ directions vanish:

$$
k^{\mu} \mathcal{G}_{\mu \nu}=l^{\mu} \mathcal{G}_{\mu \nu}=0 .
$$

This states that the gravitational field is transversal to the two isometric directions. Note that the constant $m=\frac{1}{T_{0}} \frac{\delta S_{W}}{\delta\left(\partial X^{9}\right)}$ is again the momentum of the wave in the $X^{9}$ direction, a fact that identifies the $X^{9}$ isometric direction with the direction of propagation of the wave. The other isometric direction, $Z$, is however not physical, but just an artifact of the T-duality transformation. We are therefore describing waves which are smeared in the $Z$-direction. We will see below that we can in fact rewrite $(2.20)$ as a dimensional reduction along the $Z$ direction. One can then restore the $Z$ dependence by simply undoing the reduction.

On the other hand, $\tilde{\mathcal{F}}$ is an invariant field strength, defined in terms of the T-dual of the transverse direction $Z$, that here plays the role of an extra world volume scalar [24], and the contraction of the NS-NS 2-form with the Killing vector $l^{\mu},\left(\mathrm{i}_{l} B\right)_{\mu}=l^{\nu} B_{\nu \mu}=B_{z \mu}$ :

$$
\tilde{\mathcal{F}} \equiv \partial Z+\left(\mathrm{i}_{l} B\right)_{\mu} \mathcal{D} X^{\mu} .
$$

Finally, in our notation $\mathrm{i}_{k} \mathrm{i}_{l} B \equiv B_{z x}$.

The action (2.20) is a complicated expression, which is however equivalent to the action for the massless ten-dimensional particle. We can see this explicitly by performing a Legendre transformation from $P_{9}$ to $\partial X^{9}$, for which we first rewrite $(2.20)$ as:

$$
\begin{aligned}
S_{W}\left[P_{9}\right]= & -\int d \tau P_{9}\left\{|l|\left|k^{2} l^{2}-(k . l)^{2}+\left(\mathrm{i}_{k} \mathrm{i}_{l} B\right)^{2}\right|^{-1 / 2} .\right. \\
& \cdot \sqrt{\left|\mathcal{D} X^{\mu} \mathcal{D} X^{\nu} g_{\mu \nu}+\frac{k^{2}-(k . l)^{2} / l^{2}}{k^{2} l^{2}-(k . l)^{2}+\left(\mathrm{i}_{k} \mathrm{i}_{l} B\right)^{2}} \tilde{\mathcal{F}}^{2}\right|} \\
& \left.+A^{(1)}-\partial X^{9}+\frac{\mathrm{i}_{k} \mathrm{i}_{l} B}{k^{2} l^{2}-(k . l)^{2}+\left(\mathrm{i}_{k} \mathrm{i}_{l} B\right)^{2}} \tilde{\mathcal{F}}\right\},
\end{aligned}
$$

where integration over $X^{9}$ fixes $P_{9}$ to a constant. Now we introduce an auxiliary metric $\gamma$ on the world line and define the action $S_{W}\left[P_{9}, \gamma\right]$ as

$$
\begin{aligned}
S_{W}\left[P_{9}, \gamma\right]=-\frac{m T_{0}}{2} \int d \tau \sqrt{|\gamma|} & \left\{\gamma^{-1}\left(\mathcal{D} X^{\mu} \mathcal{D} X^{\nu} g_{\mu \nu}+\frac{k^{2}-(k . l)^{2} / l^{2}}{k^{2} l^{2}-(k . l)^{2}+\left(\mathrm{i}_{k} \mathrm{i}_{l} B\right)^{2}} \tilde{\mathcal{F}}^{2}\right)\right. \\
& \left.+\frac{l^{2}}{k^{2} l^{2}-(k \cdot l)^{2}+\left(\mathrm{i}_{k} \mathrm{i}_{l} B\right)^{2}}\left(\frac{P_{9}}{m T_{0}}\right)^{2}\right\} \\
+ & +\int d \tau P_{9}\left(\partial X^{9}-A^{(1)}-\frac{\mathrm{i}_{k} \mathrm{i}_{l} B}{k^{2} l^{2}-(k . l)^{2}+\left(\mathrm{i}_{k} \mathrm{i}_{l} B\right)^{2}} \tilde{\mathcal{F}}\right)
\end{aligned}
$$


such that (2.25) is recovered upon integration on $\gamma$. The Legendre transformation defined by (2.17) gives rise to:

$$
S[\gamma]=-\frac{m T_{0}}{2} \int d \tau \sqrt{|\gamma|} \gamma^{-1}\left\{\mathcal{D} X^{\mu} \mathcal{D} X^{\nu} g_{\mu \nu}+l^{-2}\left(\partial Z+\left(i_{l} B\right)_{\mu} \partial X^{\mu}\right)^{2}\right\}
$$

which is the dimensional reduction of the action (2.18), for the ten-dimensional massless particle, along the $Z$ direction, according to the T-duality transformed reduction rules 25]

$$
g_{z z}=l^{-2}, \quad g_{z a}=l^{-2} B_{z a}, \quad g_{a b}^{(10)}=g_{a b}^{(9)}-l^{-2} B_{z a} B_{z b}
$$

where $\mu=(a, z)$ and $a=0,1, \ldots, 8$.

We have thus shown that the action (2.20), derived using T-duality, describes indeed a massless particle, though delocalised in the $Z$ direction. We will see that multiple Type IIB waves can be described by a non-Abelian extension of (2.20), but that this action cannot however be related, at least in a simple way, to some non-Abelian extension of (2.18), where the dependence on the isometric directions has been restored. We will discuss this point further in section 4 .

As we did before, we now compute, for future reference, the expansion of the action (2.20) up to linear order in the background fields. We find

$$
\begin{aligned}
& S_{W_{B}}^{\operatorname{lin}}=-m T_{0} \int d \tau\left\{1-\frac{1}{2} \dot{X}^{2}-\frac{1}{2} \dot{Z}^{2}-\frac{1}{2} h_{00}-\frac{1}{4} h_{00} \dot{X}^{2}-\frac{1}{4} h_{00} \dot{Z}^{2}-h_{0 i} \dot{X}^{i}-\frac{1}{2} h_{i j} \dot{X}^{i} \dot{X}^{j}\right. \\
& \left.-\frac{1}{2} h_{x x}+\frac{1}{4} h_{x x} \dot{X}^{2}+\frac{1}{4} h_{x x} \dot{Z}^{2}+h_{0 x}+h_{i x} \dot{X}^{i}+\frac{1}{2} h_{z z} \dot{Z}^{2}-B_{z 0} \dot{Z}+B_{z x} \dot{Z}-B_{z i} \dot{X}^{i} \dot{Z}+\ldots\right\} .
\end{aligned}
$$

Here $i, j=1, \ldots, 7$ and $\dot{X}^{2} \equiv \eta_{i j} \dot{X}^{i} \dot{X}^{j}$

\section{$3 \quad$ Matrix string theory and multiple gravitational waves}

Our starting point for the study of the effective actions associated to multiple gravitational waves is the action describing the linear couplings to background fields of Type IIA Matrix string theory in a weakly curved background. This action has been obtained in [8] and [9] from Matrix theory in a weakly coupled background [3, 26], by applying a duality chain involving T-duality and the 9-11 flip.

In the notation of [9], the Matrix string theory action for weakly coupled backgrounds is given by

$$
\begin{aligned}
S_{\mathrm{MST}}= & \frac{1}{2 \pi \beta^{2}} \int d \tau d \sigma \operatorname{STr}\left\{\frac{1}{2}\left[h_{a b}-\eta_{a b}\left(\phi-\frac{1}{2} h_{99}\right)\right] I_{h}^{a b}+2 B_{a 9} I_{s}^{a 9}+\frac{1}{2}\left[\phi+\frac{1}{2} h_{99}\right] I_{h}^{99}\right. \\
& -\frac{1}{2}\left[\phi-\frac{3}{2} h_{99}\right] I_{\phi}+C_{a}^{(1)} I_{h}^{a 9}-C_{a b 9}^{(3)} I_{s}^{a b}-C_{9}^{(1)} I_{0}^{9}-h_{a 9} I_{0}^{a}+3 B_{a b} I_{2}^{a b 9} \\
& +C_{a b c}^{(3)} I_{2}^{a b c}+\frac{1}{12} C_{a_{1} \ldots a_{4} 9}^{(5)} I_{4}^{a_{1} \ldots a_{4} 9}+\frac{1}{60} \tilde{B}_{a_{1} \ldots a_{5} 9} I_{4}^{a_{1} \ldots a_{5}}+\frac{1}{48} N_{a_{1} \ldots a_{6} 9} I_{6}^{a_{1} \ldots a_{6} 9} \\
& \left.-\frac{1}{336} N_{a_{1} \ldots a_{7} 9}^{(8)} I_{6}^{a_{1} \ldots a_{7}}\right\}
\end{aligned}
$$


where $\beta=R / \sqrt{\alpha^{\prime}}, R$ is the radius of the eleventh dimension in Matrix theory, and the ten spacetime indices are split into $a=(0, \ldots, 8)$ and 9 , the latter being the direction in which the Matrix strings are oriented.

The currents $I_{h}, I_{s}, I_{\phi}$ and $I_{p}$ give the D0-brane couplings to, respectively, the metric $h_{\mu \nu}$, the Kalb-Ramond form $B_{\mu \nu}$, the dilaton $\phi$ and the R-R fields $C^{(p+1)}$ [3, 26], and can be related to the currents appearing in the linear Matrix theory action?. However, in (3.1) they couple to different background fields because of the duality chain involved in the construction of the action [8, 9]. These currents are functions of the $U(N)$ matrix valued embedding scalars $X^{i}(i=1, \ldots, 8)$, their $U(N)$-covariant derivatives $D_{\alpha} X^{i}=\partial_{\alpha} X^{i}+i\left[A_{\alpha}, X^{i}\right]$, their commutators $\left[X^{i}, X^{j}\right]$ and the Born-Infeld vector $A_{\alpha}$. We have included in Appendix A the explicit expressions for the currents that will be of relevance to this paper. We refer to [9] for the expressions of all other currents in our notation.

In the action (3.1), $\tilde{B}$ denotes the NS-NS 6-form potential, $N^{(7)}$ is the field that couples minimally to the Type IIA Kaluza-Klein monopole, and $N^{(8)}$ couples to the so-called $\left(6,1^{2} ; 3\right)$ brane, an "exotic" brane (not predicted by the Type IIA spacetime supersymmetry algebra), first mentioned in [29, 30, 31] $\mathrm{l}$. These fields are related to the R-R 6- and 8-form potentials through the 9-11 flip:

$$
C_{a_{1} \ldots a_{6}}^{(6)} \rightarrow N_{a_{1} \ldots a_{6} 9}^{(7)}, \quad C_{a_{1} \ldots a_{7} 9}^{(8)} \rightarrow-N_{a_{1} \ldots a_{7} 9}^{(8)}
$$

In the limit $g_{s} \rightarrow 0$, the Born-Infeld vector and all R-R fields decouple from the action (3.1) and one is forced into a space of commuting matrices. In this way light-cone gauge string theory in a weakly coupled background is recovered [9]. This gives weight to the argument that the Matrix string action can indeed be derived from Matrix theory in a weakly coupled background via a duality chain.

However, a different picture emerges if one goes to the static gauge, where one identifies $X^{0}$ with the time-like world sheet coordinate $\tau$ and $X^{9}$ with the spatial world sheet coordinate $\sigma$. We also take all other embedding scalars independent of $\sigma$,

$$
X^{0}=\tau, \quad X^{9}=\sigma, \quad \partial_{\sigma} X^{a}=0 .
$$

We will refer to these conditions as static gauge conditions, though the last one represents in fact an additional truncation?.

The action (3.1) is such that the 9th direction appears as a special isometric direction on which neither the background fields nor the currents depend. This is translated into a reduced, $S O(8)$ transverse rotationally invariant action. We can however rewrite (3.1), in the static gauge, in terms of ten-dimensional pull-backs into a one-dimensional world volume, by using the techniques of gauged sigma models. If $k^{\mu}$ is the Killing vector pointing along the $X^{9}$, or $x$, direction, $k^{\mu}=\delta_{x}^{\mu}$, we can achieve invariance under the local isometric transformations generated by $k^{\mu}$,

$$
\delta X^{\mu}=\Lambda(\tau) k^{\mu},
$$

\footnotetext{
${ }^{4}$ More precise expressions for the Matrix theory couplings, to all orders in both derivatives of the background fields and the fermionic coordinates, were constructed in [27, 28] by dimensional reduction of the elevendimensional supermembrane vertex operators.

${ }^{5}$ We will choose $A_{0}=0$ throughout the paper, at the expense of losing explicit gauge invariance.

${ }^{6}$ We have included an Appendix which collects some properties of these exotic branes of interest to our work. See Appendix B for the notation and the duality relations with the branes predicted by the spacetime supersymmetry algebras.

${ }^{7}$ We thank the referee for this remark.
} 
by introducing gauge covariant derivatives, as in (2.12)

$$
\mathcal{D} X^{\mu}=D X^{\mu}-k^{-2} k_{\rho} D X^{\rho} k^{\mu},
$$

only now in terms of $U(N)$-covariant derivatives $D X^{\mu}$. In the linear approximation, the gauge covariant derivatives (3.5) reduce to $\mathcal{D} X^{\mu}=D X^{\mu}$, for $\mu \neq x$ and $\mathcal{D} X^{9}=-h_{a x} D X^{a}$. Using gauge covariant pull-backs, constructed with these gauge covariant derivatives, it is possible to eliminate the pull-back of the isometric coordinate, and to reproduce the isometric couplings in the action (3.1) in a manifestly covariant way. At the linearised level this is easy to see, given that $\mathcal{D} X^{9}$ is proportional to the linear correction to the flat metric and therefore does not contribute to the pull-back to linear order. For example, for a 1-form background field $\Sigma_{\mu}$ we have

$$
P[\Sigma]=\Sigma_{\mu} \mathcal{D} X^{\mu}=\Sigma_{0}+\beta \Sigma_{i} \dot{X}^{i},
$$

with $i=1, \ldots, 8$.

Using gauge covariant pull-backs, we are thus able to write the action (3.1) in static gauge in a manifestly covariant way, as a gauged sigma model, with the gauge covariant derivatives $\mathcal{D} X^{\mu}$ gauging away the ninth direction, which is interpreted as an isometric embedding scalar.

Filling in the expressions for the R-R currents as given in Appendix A, we obtain:

$$
\begin{aligned}
C_{9}^{(1)} I_{0}^{9} & =g_{s} \beta P\left[\mathrm{i}_{k} C^{(1)}\right] \wedge F \\
h_{a 9} I_{0}^{a} & =P\left[\mathrm{i}_{k} h\right] \\
B_{a b} I_{2}^{a b 9} & =\frac{i \beta^{2}}{3} P\left[\left(\mathrm{i}_{X} \mathrm{i}_{X}\right) B\right] \wedge F-\frac{i \beta}{3} P\left[\mathrm{i}_{[A, X]} B\right], \\
C_{a b c}^{(3)} I_{2}^{a b c} & =\frac{i \beta}{g_{s}} P\left[\left(\mathrm{i}_{X} \mathrm{i}_{X}\right) C^{(3)}\right], \\
C_{a_{1} \ldots a_{4} 9}^{(5)} I_{4}^{a_{1} \ldots a_{4} 9} & =-\frac{6 \beta^{3}}{g_{s}} P\left[\left(\mathrm{i}_{X} \mathrm{i}_{X}\right)^{2} \mathrm{i}_{k} C^{(5)}\right] \wedge F+\frac{12 \beta^{2}}{g_{s}} P\left[\left(\mathrm{i}_{X} \mathrm{i}_{X}\right) \mathrm{i}_{[A, X]} \mathrm{i}_{k} C^{(5)}\right], \\
\tilde{B}_{a_{1} \ldots a_{5} 9} I_{4}^{a_{1} \ldots a_{5}} & =\frac{30 \beta^{2}}{g_{s}^{2}} P\left[\left(\mathrm{i}_{X} \mathrm{i}_{X}\right)^{2} \mathrm{i}_{k} \tilde{B}\right], \\
N_{a_{1} \ldots a_{6} 9}^{(7)} I_{6}^{a_{1} \ldots a_{6} 9} & =-\frac{8 i \beta^{4}}{g_{s}^{2}} P\left[\left(\mathrm{i}_{X} \mathrm{i}_{X}\right)^{3} \mathrm{i}_{k} N^{(7)}\right] \wedge F+\frac{24 i \beta^{3}}{g_{s}^{2}} P\left[\left(\mathrm{i}_{X} \mathrm{i}_{X}\right)^{2} \mathrm{i}_{[A, X]} \mathrm{i}_{k} N^{(7)}\right], \\
N_{a_{1} \ldots a_{7} 9}^{(8)} I_{6}^{a_{1} \ldots a_{7}} & =\frac{56 i \beta^{3}}{g_{s}^{3}} P\left[\left(\mathrm{i}_{X} \mathrm{i}_{X}\right)^{3} \mathrm{i}_{k} N^{(8)}\right],
\end{aligned}
$$

where $F=\partial A\left(A \equiv A_{\sigma}\right)$ and

$$
\left(\mathrm{i}_{X} \mathrm{i}_{X} \Sigma\right)_{\mu_{1} \ldots \mu_{p}} \equiv X^{j} X^{i} \Sigma_{i j \mu_{1} \ldots \mu_{p}}, \quad\left(\mathrm{i}_{[A, X]} \Sigma\right)_{\mu_{1} \ldots \mu_{p}} \equiv\left[A, X^{i}\right] \Sigma_{i \mu_{1} \ldots \mu_{p}} .
$$

The inclusion involving the $A$ field is inherited from the $D_{\sigma} X^{i}$-terms in the pull-backs, of which in the static gauge only the commutator part survives. Note also that $k^{\mu}$ appears explicitly in some terms, projecting the background fields on the isometric direction:

$$
\left(\mathrm{i}_{k} \Sigma\right)_{\mu_{1} \ldots \mu_{p}} \equiv k^{\rho} \Sigma_{\rho \mu_{1} \ldots \mu_{p}}=\Sigma_{x \mu_{1} \ldots \mu_{p}} .
$$

In this manner, when taking the pull-backs to the world volume, the contribution of $X^{9}$ vanishes automatically. 
The $\sigma$-component of the Born-Infeld vector $A$ has now obtained the interpretation of a world volume scalar. This world volume scalar is associated to D0-branes "ending on the waves". Indeed, acting with the 9-11 flip on the Born-Infeld field strength of the D-string, we obtain the following invariant field strength associated to the world volume scalar $A$ :

$$
\mathcal{F}=\partial A+\frac{1}{g_{s}} P\left[C^{(1)}\right]=F+\frac{1}{g_{s}} P\left[C^{(1)}\right],
$$

since under the 9-11 flip $B_{\mu 9} \rightarrow-C_{\mu}^{(1)}$ (see [9]) $)^{\wp}$. From this invariant field strength only $F$ contributes to linear order.

Substituting (3.7)-(3.14) in the action (3.1) we obtain the following expression for the Chern-Simons action:

$$
\begin{aligned}
S_{W_{A}}^{\mathrm{CS}}= & \frac{1}{\beta^{2}} \int d \tau \mathrm{S} \operatorname{Sr}\left\{-g_{s} \beta P\left[\mathrm{i}_{k} C^{(1)}\right] \wedge F-P\left[\mathrm{i}_{k} h\right]+\frac{i \beta}{g_{s}} P\left[\left(\mathrm{i}_{X} \mathrm{i}_{X}\right) C^{(3)}\right]\right. \\
& +i \beta^{2} P\left[\left(\mathrm{i}_{X} \mathrm{i}_{X}\right) B\right] \wedge F-i \beta P\left[\mathrm{i}_{[A, X]} B\right]+\frac{\beta^{2}}{2 g_{s}^{2}} P\left[\left(\mathrm{i}_{X} \mathrm{i}_{X}\right)^{2} \mathrm{i}_{k} \tilde{B}\right] \\
& -\frac{\beta^{3}}{2 g_{s}} P\left[\left(\mathrm{i}_{X} \mathrm{i}_{X}\right)^{2} \mathrm{i}_{k} C^{(5)}\right] \wedge F+\frac{\beta^{2}}{g_{s}} P\left[\left(\mathrm{i}_{X} \mathrm{i}_{X}\right) \mathrm{i}_{[A, X]} \mathrm{i}_{k} C^{(5)}\right] \\
& -\frac{i \beta^{4}}{6 g_{s}^{2}} P\left[\left(\mathrm{i}_{X} \mathrm{i}_{X}\right)^{3} \mathrm{i}_{k} N^{(7)}\right] \wedge F+\frac{i \beta^{3}}{2 g_{s}^{2}} P\left[\left(\mathrm{i}_{X} \mathrm{i}_{X}\right)^{2} \mathrm{i}_{[A, X]} \mathrm{i}_{k} N^{(7)}\right] \\
& \left.-\frac{i \beta^{3}}{6 g_{s}^{3}} P\left[\left(\mathrm{i}_{X} \mathrm{i}_{X}\right)^{3} i_{k} N^{(8)}\right]\right\} .
\end{aligned}
$$

We claim that this action gives the Chern-Simons couplings in the world volume effective action of a system of coincident waves carrying momentum along the $X^{9}$ direction. This action is valid for weakly coupled background fields, since this is the approximation used in the Matrix theory calculation. It might seem strange at first sight that Matrix string theory can be used to describe gravitational waves. However, recall that Matrix string theory describes strings with non-zero light cone momentum, with the winding degrees of freedom entering only non-perturbatively in the description, and that string states with non-zero momentum are effectively described in terms of massless particles. It is then not surprising that the Matrix string action provides, in the static gauge, an effective description for multiple gravitational waves.

We have a number of arguments to support this claim. First of all, the monopole term $P\left[\mathrm{i}_{k} h\right]$ in (3.18) is just the linearised expression for $A-\partial X^{9}$ in (2.11), indicating that we are dealing with waves with a momentum in the $X^{9}$ direction. As we have reviewed in section 2 , waves carrying momentum in a compactified direction are T-dual to fundamental strings wound around this direction. Indeed, in [9] the non-Abelian action describing multiple Type IIB fundamental strings with non-zero winding number was derived from our action (3.1) using T-duality along the $X^{9}$ direction, which we claim is the direction of propagation of the waves. Let us also stress that we find agreement between (3.18) and the terms in the action for multiple gravitational waves that were constructed in [19]. These observations support our claim that Type IIA Matrix strings reduce to multiple waves in the static gauge.

\footnotetext{
${ }^{8}$ Note that, although we denote as well the transformed world volume field by $A$, it has a different gauge transformation rule than the original $A_{\sigma}=A$, since the gauge transformation parameters also change under the 9-11 flip.
} 
There are also more quantitative arguments supporting this identification. While in the limit $g_{s} \rightarrow 0$, (3.1) in the light-cone frame reduces to the action of light-cone gauge string theory in weakly coupled background fields [9], in the static gauge the limit $g_{s} \rightarrow 0$ reduces (3.1) to the linearised effective action of a set of commuting gravitational waves. The only non-vanishing currents, up to order $\beta^{2}$, are

$$
\begin{aligned}
I_{h}^{00} & =\mathbb{1}+\frac{1}{2} \beta^{2} \dot{X}^{2}, & I_{\phi} & =\mathbb{1}-\frac{1}{2} \beta^{2} \dot{X}^{2}, \\
I_{h}^{0 i} & =\beta \dot{X}^{i}, & I_{0}^{0} & =\mathbb{1}, \\
I_{h}^{i j} & =\beta^{2} \dot{X}^{i} \dot{X}^{j}, & I_{0}^{i} & =\beta \dot{X}^{i},
\end{aligned}
$$

and (3.1) reduces to

$$
\begin{aligned}
S_{W_{A}}^{\text {linear }}=\frac{1}{\beta^{2}} \int d \tau\{[ & \left.\frac{1}{2} h_{00}-h_{0 x}+\frac{1}{2} h_{x x}\right] \mathbb{1}+\beta\left[\left(h_{0 i}-h_{i x}\right) \dot{X}^{i}\right] \\
& \left.+\frac{1}{2} \beta^{2}\left[h_{i j} \dot{X}^{i} \dot{X}^{j}+\frac{1}{2}\left(h_{00}-h_{x x}\right) \dot{X}^{2}\right]+\mathcal{O}\left(\beta^{3}\right)\right\} .
\end{aligned}
$$

This action coincides, up to the relative rescalings between the Abelian and Matrix theory calculations, with the linear order contribution to the expansion of the effective action (2.11), for Abelian gravitational waves, given by (2.19).

Finally, let us point out that it is also possible to take the Abelian limit without taking $g_{s} \rightarrow 0$, in which case we get linearised non-perturbative corrections to the action (2.11). Substituting the expressions for the NS-NS currents (see Appendix A) in (3.1), together with (3.7), we find:

$$
S_{W_{A}}^{\text {corr }}=\frac{g_{s}}{\beta} \int d \tau\left\{P\left[\mathrm{i}_{k} C^{(1)}\right] \wedge F+\beta C_{i}^{(1)} \dot{A} \dot{X}^{i}\right\}
$$

One can easily see that the Legendre transformation of section 2 restores the $X^{9}$ dependence also in these correcting terms, giving rise in (2.18) to an extra linear coupling

$$
S[\gamma]=-\frac{m T_{0}}{2} \int d \tau \sqrt{|\gamma|} \gamma^{-1}\left\{\partial X^{\mu} \partial X^{\nu} g_{\mu \nu}-\frac{g_{s}}{2} C_{\mu}^{(1)} \partial X^{\mu} F\right\} .
$$

Identifying the full couplings whose linear expansion gives rise to this contribution is not easy without the help of some additional requirement. We will discuss in the next section that it is possible in principle to identify some of these couplings by imposing S-duality invariance for Type IIB waves, and then T-dualising.

We have thus discussed that the Matrix theory calculation describes Type IIA waves in terms of a non-Abelian extension of the action (2.11), for which the direction of propagation of the waves appears as a special isometric direction. One could try now to restore the dependence on $X^{9}$, by performing a Legendre transformation along the lines of section 2 . This procedure, however, does not work, as it can easily be argued. First, the direction of propagation of the waves, as it arises in the calculation, is not matrix-valued, since it is identified with the spatial world sheet direction of D-strings (see [9]). So, even though we can in principle restore some explicit dependence on $\partial X^{9}$, the new terms are going to be Abelian. Moreover, it is clear that the Legendre transformation cannot give rise to nonAbelian commutators of the transverse scalars involving $X^{9}$. This results in an action with, still, $S O(8)$ transverse rotational invariance. 
In fact, the existence of a compact isometric direction gives the spacetime on which the waves propagate a non-trivial topology. This compact direction allows $p$-branes to wind around it or live in its transverse space, giving rise to the specific dielectric couplings in (3.18), for which the $k^{\mu}$ dependence cannot be eliminated. These extra topologically nontrivial couplings are in a sense analogous to the supergravity solutions of [32], corresponding to $p$-branes wound around different compact dimensions.

\section{Multiple Type IIB gravitational waves}

In this section we present the action associated to multiple Type IIB gravitational waves. We calculate it by performing a T-duality transformation, along a transverse direction, on the Matrix string theory action with linear couplings to closed string fields, given by (3.1). We argue that this action describes Type IIB waves in the static gauge since, as we have seen, Matrix string theory describes Type IIA waves in this gauge, and we are T-dualising along a transverse direction (see Figure 1). The action thus obtained contains the linear couplings of multiple Type IIB gravitons to closed string fields.

\subsection{The action for multiple Type IIB gravitational waves}

Since we are working with actions which are only linear in the background fields, it is sufficient to use the T-duality transformation rules to linear order. For a T-duality transformation in a generic $y$ direction, these are given by:

$$
\begin{array}{lll}
h_{y y} \rightarrow-h_{y y}, & B_{a b} \rightarrow B_{a b}, & N_{a_{1} \ldots a_{6} y}^{(7)} \rightarrow \tilde{B}_{a_{1} \ldots a_{6}}, \\
h_{a y} \rightarrow-B_{a y}, & \tilde{B}_{a_{1} \ldots a_{5} y} \rightarrow \tilde{B}_{a_{1} \ldots a_{5} y}, & \left(i_{k} N^{(7)}\right)_{a_{1} \ldots a_{5} y} \rightarrow\left(i_{k} N^{(7)}\right)_{a_{1} \ldots a_{5} y}, \\
h_{a b} \rightarrow h_{a b}, & \tilde{B}_{a_{1} \ldots a_{6}} \rightarrow \mathcal{N}_{a_{1} \ldots a_{6} y}^{(7)}, & \left(i_{k} N^{(7)}\right)_{a_{1} \ldots a_{6}} \rightarrow\left(i_{k} \mathcal{N}^{(8)}\right)_{a_{1} \ldots a_{6} y}, \\
\phi \rightarrow \phi-\frac{1}{2} h_{y y}, & C_{a_{1} \ldots a_{p-1} y}^{(p)} \rightarrow C_{a_{1} \ldots a_{p-1}}^{(p-1)}, & \left(i_{k} N^{(8)}\right)_{a_{1} \ldots a_{6} y} \rightarrow\left(i_{k} N^{(8)}\right)_{a_{1} \ldots a_{6} y}, \\
B_{a y} \rightarrow-h_{a y}, & C_{a_{1} \ldots a_{p}}^{(p)} \rightarrow C_{a_{1} \ldots a_{p} y}^{(p+1)}, & \left(i_{k} N^{(8)}\right)_{a_{1} \ldots a_{7}} \rightarrow\left(i_{k} N^{(9)}\right)_{a_{1} \ldots a_{7} y} .
\end{array}
$$

We see that for higher order background fields T-duality gives rise to new gravitational fields, $N^{(p)}$ and $\mathcal{N}^{(q)}$, which have in fact already been encountered in the literature [24, 33]. We have summarised the notation and the duality properties of these fields in Appendix B. The fields $N^{(7)}$ and $\mathcal{N}^{(7)}$ couple minimally to Type IIB Kaluza-Klein monopoles with different TaubNUT directions, as we will clarify later on in this section. $N^{(8)}, \mathcal{N}^{(8)}$ and $N^{(9)}$ couple to different "exotic" branes, not predicted by the Type IIB spacetime supersymmetry algebra?"

Applying T-duality to the action (3.1) in a transverse direction $Z$ we obtain the following

\footnotetext{
${ }^{9} \mathrm{~A}$ more detailed explanation of the role played by these branes is contained in Appendix B.
} 
linear action:

$$
\begin{aligned}
& S_{W_{B}}^{\text {linear }}=\frac{1}{2 \pi \beta^{2}} \int d \tau d \sigma \operatorname{STr}\left\{\frac{1}{2}\left[h_{a b}-\eta_{a b}\left(\phi-\frac{1}{2} h_{z z}-\frac{1}{2} h_{99}\right)\right] I_{h}^{a b}-B_{a z} I_{h}^{a z}\right. \\
& \quad-\frac{1}{2}\left[\phi+\frac{1}{2} h_{z z}-\frac{1}{2} h_{99}\right] I_{h}^{z z}+C_{a z}^{(2)} I_{h}^{a 9}+\frac{1}{2}\left[\phi-\frac{1}{2} h_{z z}+\frac{1}{2} h_{99}\right] I_{h}^{99}+C^{(0)} I_{h}^{z 9} \\
& \quad-\frac{1}{2}\left[\phi-\frac{1}{2} h_{z z}-\frac{3}{2} h_{99}\right] I_{\phi}+C_{a b z 9}^{(4)} I_{s}^{a b}+2 C_{a 9}^{(2)} I_{s}^{a z}+2 B_{a 9} I_{s}^{a 9}+2 h_{z 9} I_{s}^{z 9} \\
& \quad-h_{a 9} I_{0}^{a}-B_{z 9} I_{0}^{z}+C_{z 9}^{(2)} I_{0}^{9}+C_{a b c z}^{(4)} I_{2}^{a b c}+3 C_{a b} I_{2}^{a b z}+3 B_{a b} I_{2}^{a b 9}-6 h_{a z} I_{2}^{a z 9} \\
& \quad+\frac{1}{60} \mathcal{N}_{a_{1} \ldots a_{5} 9 z}^{(7)} I_{4}^{a_{1} \ldots a_{5}}+\frac{1}{12} \tilde{B}_{a_{1} \ldots a_{4} z 9} I_{4}^{a_{1} \ldots a_{4} z}-\frac{1}{12} C_{a_{1} \ldots a_{4} z 9}^{(6)} I_{4}^{a_{1} \ldots a_{4} 9}-\frac{1}{3} C_{a b c 9}^{(4)} I_{4}^{a b c z 9} \\
& \left.\quad-\frac{1}{336} N_{a_{1} \ldots a_{7} 9 z}^{(9)} I_{6}^{a_{1} \ldots a_{7}}+\frac{1}{48} N_{a_{1} \ldots a_{6} 9 z}^{(8)} I_{6}^{a_{1} \ldots a_{6} z}+\frac{1}{48} \mathcal{N}_{a_{1} \ldots a_{6} 9 z}^{(8)} I_{6}^{a_{1} \ldots a_{6} 9}+\frac{1}{8} N_{a_{1} \ldots a_{5} z 9}^{(7)} I_{6}^{a_{1} \ldots a_{5} z 9}\right\}
\end{aligned}
$$

Here now, the indices $a$ run from 0 to 7 . As a consistency check we have derived the same action by performing a T-duality transformation in the action describing multiple Type IIA fundamental strings, in the static gauge, constructed in [9], this time along the spatial world volume direction of the strings (see Figure 1). We recover (4.2) up to a sign difference for the R-R fields. Note, however, that this sign difference is not physical. T-duality transformations in two distinct directions only commute up to a sign in the right moving R-sector 34] $\left(T_{1} \cdot T_{2}=\right.$ $(-)^{F_{R}} T_{2} \cdot T_{1}$ ), which changes the sign of all the R-R fields. However, $(-)^{F_{R}}$ is a symmetry of the theory, and this makes this sign difference unphysical.

In analogy with the linear action (3.1), we can argue that the action (4.2) describes multiple light-cone fundamental strings in a weakly coupled Type IIB background. Our main interest here, however, lays in the wave interpretation of the action (4.2), so that we take, instead, the static gauge (3.3).

In this gauge we obtain a one-dimensional action with two isometries: one corresponding to translations along the direction of propagation $X^{9}$ of the waves and the other to translations along the transversal direction $Z$, in which the T-duality has been performed. The Matrix theory calculation seems then to be describing Type IIB waves in terms of a nonAbelian extension of the action (2.20), as we will further check. In this action the direction of propagation of the waves appears as an isometric direction, but there is as well an extra isometry in the $Z$ direction. We are therefore dealing with multiple gravitational waves which are delocalised in the $Z$ direction. As we discussed in section 2, the isometry in $Z$ is however not physical, but just an artifact of the T-duality transformation. Indeed, for the Abelian case we showed how it is possible to restore the $Z$-dependence in the action. However, the corresponding Legendre transformation in the non-Abelian case yields still an action in which $X^{9}$ and $Z$ appear as special directions (see the discussion at the end of the previous section). We are thus constrained to work, in the non-Abelian case, with an action with two isometries, and assume the presence of the extra, unphysical isometry.

As we did in the previous section, we can rewrite the Matrix theory action (4.2) in a manifestly covariant way as a gauged sigma model. Introducing Killing vectors associated to the two isometries $k^{\mu}=\delta_{x}^{\mu}$ and $l^{\mu}=\delta_{z}^{\mu}$ and gauge covariant derivatives, we can achieve invariance under local isometric transformations generated by $k^{\mu}$ and $l^{\mu}$ :

$$
\delta X^{\mu}=\Lambda^{(1)}(\tau) k^{\mu}+\Lambda^{(2)}(\tau) l^{\mu},
$$


with the gauge covariant derivatives defined as in (2.22):

$$
\mathcal{D} X^{\mu}=D X^{\mu}-\frac{l^{2}\left(k_{\rho} D X^{\rho}\right)-(k \cdot l)\left(l_{\rho} D X^{\rho}\right)}{k^{2} l^{2}-(k \cdot l)^{2}} k^{\mu}-\frac{k^{2}\left(l_{\rho} D X^{\rho}\right)-(k \cdot l)\left(k_{\rho} D X^{\rho}\right)}{k^{2} l^{2}-(k \cdot l)^{2}} l^{\mu},
$$

now in terms of $U(N)$-covariant derivatives $D X^{\mu}$. In the linear approximation, $\mathcal{D} X^{\mu}=D X^{\mu}$ for $\mu \neq x, z$, whereas $\mathcal{D} X^{9}=-h_{a x} D X^{a}$ and $\mathcal{D} Z=-h_{a z} D X^{a}$. Therefore, at the linearised level, $\mathcal{D} X^{9}$ and $\mathcal{D} Z$ do not contribute to the pull-backs.

Filling in the expressions for the R-R currents as given in Appendix A, we can then write the Chern-Simons part of (4.2), in the static gauge, in a manifestly covariant way, as:

$$
\begin{aligned}
& S_{W_{B}}^{\mathrm{CS}}=\frac{1}{\beta^{2}} \int d \tau \operatorname{STr}\left\{-P\left[\mathrm{i}_{k} h\right]-i g_{s} \beta[A, \omega] P\left[\mathrm{i}_{l} h\right]-i g_{s} \beta^{2} P\left[\mathrm{i}_{[\omega, X]} \mathrm{i}_{l} h\right] \wedge F\right. \\
& +i g_{s} \beta^{2} P\left[\mathrm{i}_{[A, X]} \mathrm{i}_{l} h\right] \wedge \tilde{F}+g_{s} \beta P\left[\mathrm{i}_{l} \mathrm{i}_{k} B\right] \wedge \tilde{F}-i \beta P\left[\mathrm{i}_{[A, X]} B\right]+i \beta^{2} P\left[\left(\mathrm{i}_{X} \mathrm{i}_{X}\right) B\right] \wedge F \\
& -g_{s} \beta P\left[\mathrm{i}_{l} \mathrm{i}_{k} C^{(2)}\right] \wedge F-i \beta P\left[\mathrm{i}_{[\omega, X]} C^{(2)}\right]+i \beta^{2} P\left[\left(\mathrm{i}_{X} \mathrm{i}_{X}\right) C^{(2)}\right] \wedge \tilde{F}-\frac{i \beta}{g_{s}} P\left[\left(\mathrm{i}_{X} \mathrm{i}_{X}\right) \mathrm{i}_{l} C^{(4)}\right] \\
& +\beta^{2} P\left[\mathrm{i}_{[A, X]} \mathrm{i}_{[\omega, X]} \mathrm{i}_{k} C^{(4)}\right]-\beta^{2}[A, \omega] P\left[\left(\mathrm{i}_{X} \mathrm{i}_{X}\right) \mathrm{i}_{k} C^{(4)}\right]-\beta^{3} P\left[\mathrm{i}_{[A, X]}\left(\mathrm{i}_{X} \mathrm{i}_{X}\right) \mathrm{i}_{k} C^{(4)}\right] \wedge \tilde{F} \\
& -\beta^{3} P\left[\mathrm{i}_{[\omega, X]}\left(\mathrm{i}_{X} \mathrm{i}_{X}\right) i_{k} C^{(4)}\right] \wedge F+\frac{\beta^{2}}{g_{s}} P\left[\mathrm{i}_{[A, X]}\left(\mathrm{i}_{X} \mathrm{i}_{X}\right) \mathrm{i}_{l} \mathrm{i}_{k} C^{(6)}\right]-\frac{\beta^{3}}{2 g_{s}} P\left[\left(\mathrm{i}_{X} \mathrm{i}_{X}\right)^{2} \mathrm{i}_{l} \mathrm{i}_{k} C^{(6)}\right] \wedge F \\
& \quad-\frac{\beta^{2}}{g_{s}} P\left[\mathrm{i}_{[\omega, X]}\left(\mathrm{i}_{X} \mathrm{i}_{X}\right) \mathrm{i}_{l} \mathrm{i}_{k} \tilde{B}\right]+\frac{\beta^{3}}{2 g_{s}} P\left[\left(\mathrm{i}_{X} \mathrm{i}_{X}\right)^{2} \mathrm{i}_{l} \mathrm{i}_{k} \tilde{B}\right] \wedge \tilde{F}-\frac{\beta^{2}}{2 g_{s}^{2}} P\left[\left(\mathrm{i}_{X} \mathrm{i}_{X}\right)^{2} \mathrm{i}_{l} \mathrm{i}_{k} \mathcal{N}^{(7)}\right] \\
& +\frac{i \beta^{3}}{g_{s}} P\left[\mathrm{i}_{[A, X]}\left(\mathrm{i}_{X} \mathrm{i}_{X}\right) \mathrm{i}_{[\omega, X]} \mathrm{i}_{l} \mathrm{i}_{k} N^{(7)}\right]-\frac{i \beta^{3}}{2 g_{s}}[A, \omega] P\left[\left(\mathrm{i}_{X} \mathrm{i}_{X}\right)^{2} \mathrm{i}_{l} \mathrm{i}_{k} N^{(7)}\right] \\
& +\frac{i \beta^{4}}{2 g_{s}} P\left[\left(\mathrm{i}_{X} \mathrm{i}_{X}\right)^{2} \mathrm{i}_{[\omega, X} \mathrm{i}_{l} \mathrm{i}_{k} N^{(7)}\right] \wedge F-\frac{i \beta^{4}}{2 g_{s}} P\left[\left(\mathrm{i}_{X} \mathrm{i}_{X}\right)^{2} \mathrm{i}_{[A, X]} \mathrm{i}_{l} \mathrm{i}_{k} N^{(7)}\right] \wedge \tilde{F} \\
& +\frac{i \beta^{3}}{2 g_{s}^{2}} P\left[\mathrm{i}_{[A, X]}\left(\mathrm{i}_{X} \mathrm{i}_{X}\right)^{2} \mathrm{i}_{l} \mathrm{i}_{k} \mathcal{N}^{(8)}\right]-\frac{i \beta^{4}}{6 g_{s}^{2}} P\left[\left(\mathrm{i}_{X} \mathrm{i}_{X}\right)^{3} \mathrm{i}_{l} \mathrm{i}_{k} \mathcal{N}^{(8)}\right] \wedge F \\
& \left.+\frac{i \beta^{3}}{2 g_{s}^{2}} P\left[\mathrm{i}_{[\omega, X]}\left(\mathrm{i}_{X} \mathrm{i}_{X}\right)^{2} \mathrm{i}_{l} \mathrm{i}_{k} N^{(8)}\right]-\frac{i \beta^{4}}{6 g_{s}^{2}} P\left[\left(\mathrm{i}_{X} \mathrm{i}_{X}\right)^{3} \mathrm{i}_{l} \mathrm{i}_{k} N^{(8)}\right] \wedge \tilde{F}+\frac{i \beta^{3}}{6 g_{s}^{3}} P\left[\left(\mathrm{i}_{X} \mathrm{i}_{X}\right)^{3} \mathrm{i}_{l} \mathrm{i}_{k} N^{(9)}\right]\right\}
\end{aligned}
$$

Like the action for Type IIA waves, this action gives the Chern-Simons couplings of a system of coincident Type IIB waves for weakly coupled background fields, due to the linear approximation of the Matrix theory calculation.

Here $F=\partial A$ and $\tilde{F}=\partial \omega$, where we have redefined the coordinate $Z$, which now plays the role of a world-volume scalar [24], as

$$
Z \equiv g_{s} \omega
$$

This rescaling will be useful when we study the behaviour of the action under S-duality. The commutator inclusion terms are defined as

$$
\left(\mathrm{i}_{[A, X]} \Sigma\right)_{\mu_{1} \ldots \mu_{p}} \equiv\left[A, X^{i}\right] \Sigma_{i \mu_{1} \ldots \mu_{p}}, \quad\left(\mathrm{i}_{[\omega, X]} \Sigma\right)_{\mu_{1} \ldots \mu_{p}} \equiv\left[\omega, X^{i}\right] \Sigma_{i \mu_{1} \ldots \mu_{p}}
$$

and $\mathrm{i}_{l} \mathrm{i}_{k} \Sigma$ denotes double contractions:

$$
\left(\mathrm{i}_{l} \mathrm{i}_{k} \Sigma\right)_{\mu_{1} \ldots \mu_{p}} \equiv l^{\lambda} k^{\rho} \Sigma_{\rho \lambda \mu_{1} \ldots \mu_{p}}=\Sigma_{x z \mu_{1} \ldots \mu_{p}} .
$$


Background fields contracted in this manner are such that both the $X^{9}$ and $Z$ contributions vanish when taking the pull-backs to the world volume .

Note that, as in the Type IIA case, the monopole term $P\left[i_{k} h\right]$ in the Chern-Simons action above is the origin of the momentum carried by the waves in the propagation direction $X^{9}$.

The action for multiple Type IIB gravitational waves that we have thus derived seems particularly involved, compared to the dielectric action of other $p$-branes. This is because we are dealing with an action that has two compact isometry directions, which give the spacetime we consider a non-trivial topology. The two compact directions allow $p$-branes to wind around them, giving rise to the different inclusion terms that characterise the action (4.5).

Note that there are two different gravitational 7 -forms that couple in the action (4.5), $N^{(7)}$ and $\mathcal{N}^{(7)}$, which emerge through the T-duality rules (4.1). These fields can couple in the Chern-Simons action (4.5) only because there exist two isometric directions. $N^{(7)}$ is electricmagnetic dual to the Killing vector $k_{\mu}$, considered as a 1 -form, and $\mathcal{N}^{(7)}$ to $l_{\mu}$. Therefore, they are associated to KK-monopoles with Taub-NUT directions pointing along the two different Killing vectors. Similarly, $N^{(8)}$ and $\mathcal{N}^{(8)}$ are Poincaré duals to the scalars $k^{2}$ and $l^{2}$ (see Appendix B).

We will now give some arguments that support the interpretation of the action (4.5) as the non-Abelian Chern-Simons action for multiple Type IIB gravitational waves. First we will show the S-duality invariance, not only of (4.5), but of the full action (4.2), in agreement with the predictions of the Type IIB supersymmetry algebra. Secondly we will show that, in analogy with the Type IIA case, we obtain in the weak coupling limit, the linearised Abelian action for a massless particle.

\subsection{S-duality invariance}

In this section we check that the action that we have proposed for multiple gravitational waves in linearised Type IIB backgrounds is manifestly S-duality invariant. This is a nontrivial check for our action, given that the S-selfduality of the (single) Type IIB gravitational wave is predicted by the analysis of the spacetime supersymmetry algebra of the theory [35]. The fact that the invariance also holds for the non-Abelian case is remarkable and an argument in favour of our interpretation.

To linear order, the S-duality rules for the metric, the dilaton and the R-R scalar are given by

$$
h_{\mu \nu} \rightarrow h_{\mu \nu}-\eta_{\mu \nu} \phi, \quad \phi \rightarrow-\phi, \quad C^{(0)} \rightarrow-C^{(0)},
$$

while the 2-form, 6-form and 8-form fields combine in S-duality doublets

$$
\begin{array}{lll}
B \rightarrow-C^{(2)}, & \tilde{B} \rightarrow-C^{(6)}, & \mathcal{N}^{(8)} \rightarrow-N^{(8)}, \\
C^{(2)} \rightarrow B, & C^{(6)} \rightarrow \tilde{B}, & N^{(8)} \rightarrow \mathcal{N}^{(8)} .
\end{array}
$$

These transformation rules for $N^{(8)}$ and $\mathcal{N}^{(8)}$ can easily be derived taking into account the origin of these fields from eleven dimensions, where S-duality is realised as a modular transformation in the 2-torus. Indeed, we easily see from Figure 3 in Appendix B, that both fields have their M-theory origin in the field that couples minimally to the Kaluza-Klein monopole, and that one gets one or the other by simply interchanging the eleventh and T-duality directions, which is well known to generate S-duality in the Type IIB theory [25].

$N^{(7)}, \mathcal{N}^{(7)}$ and $N^{(9)}$ are on the other hand singlets. This is clear for $N^{(7)}, \mathcal{N}^{(7)}$, since they couple to Type IIB Kaluza-Klein monopoles with different Taub-NUT directions, and these 
are known to be S-duality invariant [35]. One can check it explicitly by looking at the gauge transformation rules of these fields, computed in [24]. That this is also the case for $N^{(9)}$ was shown in [33].

Let us now study the S-duality transformation rules of the world volume fields. The two world volume fields $A$ and $\omega$ form a doublet under S-duality, as we can deduce by looking at the invariant field strengths they form with the pull-backs of the background fields. Tdualising the invariant field strength (3.17), that couples in the world volume of the Type IIA waves, we derive the following invariant field strength for the world volume scalar $A$ :

$$
\mathcal{F}=\partial A-\frac{1}{g_{s}} P\left[i_{l} C^{(2)}\right]
$$

The invariant field strength for $\omega$ was in turn derived in section 2, for the Abelian case. Expression (2.24) becomes $g_{s} \partial \omega+\left(\mathrm{i}_{l} B\right)_{\mu} \mathcal{D} X^{\mu}$. Therefore in our Matrix theory notation we have:

$$
\tilde{\mathcal{F}}=\partial \omega+\frac{1}{g_{s}} P\left[\mathrm{i}_{l} B\right] .
$$

We thus see that $A$ and $\omega$ are associated to D-strings and F-strings respectively, wound around the $Z$ direction, ending on the waves. Given that $B$ and $C^{(2)}$ transform as a doublet under S-duality (see (4.10)), $\omega$ and $A$ must transform as:

$$
\omega \rightarrow A, \quad A \rightarrow-\omega .
$$

With this behaviour of the background and world volume fields under S-duality, it is straightforward to check that the Chern-Simons action (4.5) is indeed invariant under Sduality.

As to the Born-Infeld part of the action (4.2), it is shown in Appendix A that, at least up to order $\beta^{2}$, the matrix currents $\left(I_{h}^{a 9}, I_{h}^{a z}\right)$ and $\left(I_{s}^{a 9}, I_{s}^{a z}\right)$ form doublets under the transformations (4.13), and that $I_{h}^{9 z}$ picks up a minus sign, while $I_{h}^{99}$ and $I_{h}^{z z}$ get mapped into each other. All other currents are left invariant.

It is not difficult to see that the Born-Infeld action, at least up to order $\beta^{2}$, is then S-duality invariant.

\subsection{The Abelian limit}

As in the previous section, we can support our claim that the current Matrix theory calculation describes Type IIB gravitational waves by taking the weak coupling limit and checking that the action (4.2) reduces to the linearised effective action for a set of commuting Type IIB gravitational waves. As we already discussed, Matrix theory describes Type IIB waves in terms of a non-Abelian extension of the action (2.20), so we should compare, in the weak coupling limit, with the linear expansion of this action, given by expression (2.29).

Again, in the limit $g_{s} \rightarrow 0$, the Born-Infeld vector and all but the NS-NS fields decouple, and we are forced into a space of commuting matrices. The expressions for the relevant 
currents can be found in Appendix A. In this limit they are given, up to order $\beta^{2}$, by

$$
\begin{array}{ll}
I_{\phi}=\mathbb{1}-\frac{1}{2} \beta^{2}\left(\dot{X}^{2}+\dot{Z}^{2}\right), & I_{h}^{i z}=\beta^{2} \dot{X}^{i} \dot{Z}, \\
I_{h}^{00}=\mathbb{1}+\frac{1}{2} \beta^{2}\left(\dot{X}^{2}+\dot{Z}^{2}\right), & I_{h}^{z z}=\beta^{2} \dot{Z}^{2}, \\
I_{h}^{0 i}=\beta \dot{X}^{i}, & I_{0}^{0}=\mathbb{1}, \\
I_{h}^{0 z}=\beta \dot{Z}, & I_{0}^{i}=\beta \dot{X}^{i}, \\
I_{h}^{i j}=\beta^{2} \dot{X}^{i} \dot{X}^{j}, & I_{0}^{z}=\beta \dot{Z},
\end{array}
$$

with all other currents equal to zero. The action (4.2) then reduces to

$$
\begin{array}{r}
S_{W_{B}}^{\text {linear }}=\frac{1}{\beta^{2}} \int d \tau\left\{\left[\frac{1}{2} h_{00}-h_{0 x}+\frac{1}{2} h_{x x}\right] \mathbb{1}+\beta\left[\left(h_{0 i}-h_{i x}\right) \dot{X}^{i}-\left(B_{0 z}+B_{z x}\right) \dot{Z}\right]\right. \\
\left.+\frac{1}{2} \beta^{2}\left[h_{i j} \dot{X}^{i} \dot{X}^{j}+\frac{1}{2}\left(h_{00}-h_{x x}\right)\left(\dot{X}^{2}+\dot{Z}^{2}\right)-h_{z z} \dot{Z}^{2}-2 B_{i z} \dot{X}^{i} \dot{Z}\right]+\mathcal{O}\left(\beta^{3}\right)\right\}
\end{array}
$$

where $\dot{X}^{2} \equiv \eta_{i j} \dot{X}^{i} \dot{X}^{j}$. Therefore we reproduce, up to rescalings, the linearised effective action (2.29) for commuting gravitational waves derived in section 2 .

If we take the Abelian limit without sending the string coupling constant to zero, we recover the action (2.20) supplemented with non-perturbative corrections associated to Dbrane degrees of freedom. These corrections are indeed necessary in order to achieve invariance of (2.20) under S-duality, as predicted by the Type IIB supersymmetry algebra. From the linearised Matrix theory calculation we find the non-perturbative corrections:

$$
\begin{array}{r}
S_{W_{B}}^{\mathrm{corr}}=\frac{g_{s}}{\beta} \int d \tau\left\{-\mathrm{i}_{l} \mathrm{i}_{k} C^{(2)} \wedge F-P\left[\mathrm{i}_{l} C^{(2)}\right] \wedge F+\beta C^{(0)} F \dot{Z}\right. \\
\left.+g_{s} \beta\left[\phi F^{2}+\frac{g_{s} \beta}{4}\left(h_{00}-h_{x x}-2 h_{z z}\right) F^{2}\right]\right\} .
\end{array}
$$

Note that the two terms in $C^{(2)}$ are precisely the S-duals of the $B$ terms in (4.15). Indeed it is easy to check that the sum of (4.15) and (4.16) is S-duality invariant.

We can derive this S-duality invariant action as the linear expansion of the following nonperturbative modification of (2.20):

$$
\begin{aligned}
S_{W_{B}}= & -m T_{0} \int d \tau\left\{|l|\left|k^{2} l^{2}-(k . l)^{2}+\left(\mathrm{i}_{k} \mathrm{i}_{l} B\right)^{2}\right|^{-1 / 2}\right. \\
& \cdot \sqrt{\left|\mathcal{D} X^{\mu} \mathcal{D} X^{\nu} g_{\mu \nu}+\frac{k^{2}-(k . l)^{2} / l^{2}}{k^{2} l^{2}-(k . l)^{2}+\left(\mathrm{i}_{k} \mathrm{i}_{l} B\right)^{2}} e^{\phi} \mathrm{F}^{T} \mathrm{MF}\right|} \\
& \left.+A^{(1)}-\partial X^{9}+\frac{\mathrm{i}_{k} \mathrm{i}_{l} B \wedge \tilde{F}-\mathrm{i}_{k} \mathrm{i}_{l} C^{(2)} \wedge F}{k^{2} l^{2}-(k . l)^{2}+\left(\mathrm{i}_{k} \mathrm{i}_{l} B\right)^{2}}\right\}
\end{aligned}
$$

Here $\mathrm{F}^{T} \mathrm{MF}$ is the $S L(2, \mathbb{R})$-invariant combination:

$$
\mathrm{F}^{T} \mathrm{MF}=\left(\begin{array}{cc}
\tilde{\mathcal{F}} & \mathcal{F}
\end{array}\right) e^{\phi}\left(\begin{array}{cc}
e^{-2 \phi}+C^{(0) 2} & C^{(0)} \\
C^{(0)} & 1
\end{array}\right)\left(\begin{array}{c}
\tilde{\mathcal{F}} \\
\mathcal{F}
\end{array}\right)
$$


with

$$
\tilde{\mathcal{F}}=\partial Z+P\left[\mathrm{i}_{l} B\right], \quad \mathcal{F}=\partial A-P\left[\mathrm{i}_{l} C^{(2)}\right],
$$

the gauge invariant curvatures of the world volume scalars $Z$ and $A$. Note that $\tilde{\mathcal{F}}$ is the field strength that coupled in the perturbative action $(2.20)$ (see $(2.24)$ ), and that $\mathcal{F}$ is its $\mathrm{S}$-dual, which has to couple as well in the non-perturbative action in order to achieve invariance under S-duality $\mathbb{D}$. The $S L(2, \mathbb{R})$-invariant combination (4.18) appears as well in the world volume effective action of the Type IIB Kaluza-Klein monopole [24], a brane which is also known to behave as a singlet under S-duality.

This extension of (4.17) is, however, not fully S-duality invariant, since we have not included the S-dual of the $\left(\mathrm{i}_{k} \mathrm{i}_{l} B\right)^{2}$ term. Since this term does not contribute to the linearised action, we cannot determine the correct S-duality invariant combination associated to it by comparing with the Matrix theory calculation. By T-dualising the full S-duality invariant action we would be able to derive as well the non-perturbative couplings in the action of Abelian Type IIA waves, whose linear expansion we determined using Matrix theory.

\section{$5 \quad$ Dielectric wave solutions}

In this section we present some explicit solutions of gravitational waves expanding into dielectric branes, rising from the non-Abelian couplings derived in the previous sections.

We have seen in sections 3 and 4 that the Matrix theory calculation captures, already to linear order, the non-perturbative degrees of freedom associated to D-brane states. These new degrees of freedom translate into correction terms, (3.21) and (4.16), to the perturbative, Abelian actions (2.11) and (2.20). In the non-Abelian case we find as well non-perturbative terms corresponding to the contraction of the background fields with the embedding scalars, yielding multipole couplings of the same type as those derived in [2, 26] for the action of coinciding D-branes. These couplings are responsible for the expansion of the waves into dielectric configurations, in the presence of external field strengths.

In [8] it was shown that the term

$$
\frac{i}{\beta g_{s}} P\left[\left(\mathrm{i}_{X} \mathrm{i}_{X}\right) C^{(3)}\right]
$$

in the action (3.18), gives rise to a potential

$$
V(X)=\operatorname{STr}\left\{-\frac{1}{4 g_{s}^{2}}[X, X]^{2}+\frac{i}{3 g_{s}} X^{k} X^{j} X^{i} F_{0 i j k}^{(4)}\right\}
$$

which has a stable solution of the form

$$
F_{0 i j k}^{(4)}=f \epsilon_{i j k}, \quad X^{i}=-\frac{1}{4} g_{s} f J^{i},
$$

where the matrices $J^{i}$ form an $N \times N$ representation of $S U(2)$ [2].

The interpretation of the Matrix string action as describing multiple Type IIA waves helped clarifying this Matrix string dielectric solution (see section 7.3 in [9]). The solution in [8] was interpreted in terms of $N$ fundamental strings expanding into a fuzzy D2-brane, with world

\footnotetext{
${ }^{10}$ The relative $1 / g_{s}$ factor between (4.19) and the field strengths given in (4.11) and 4.12 is due to the rescalings of the Matrix theory calculation, that take $\partial A \rightarrow g_{s} \partial A$ (see Appendix $\mathrm{A}$ ).
} 
volume $\tau$ times a 2 -sphere in the $(i, j, k)$-directions. However, the world volume of the D2brane does not contain the spatial world sheet direction $X^{9}$ of the string, nor can the dipole coupling $\left(\mathrm{i}_{X} \mathrm{i}_{X}\right) C^{(3)}$ in the Chern-Simons action appear as a pull-back to a two-dimensional world volume, as required in the expanding strings interpretation. Schiappa pointed out that the expanding fundamental strings were actually describing gravitons, though no explicit check of this assessment was given.

With our interpretation of Matrix string theory as describing multiple gravitational waves we can see explicitly that this solution does indeed correspond to multiple gravitons expanded into a D2-brane, which is in fact transverse to their direction of propagation $\square$ (see also [9]). Moreover, within this interpretation (5.1) can naturally be written as a pull-back to the one-dimensional world line of the waves.

Other terms in the action (3.18) describe multipole couplings of the waves to NS5-branes and KK-monopoles, although, to our knowledge, no stable dielectric solutions associated to these terms are known.

Similarly, in the Type IIB action (4.5) the dielectric coupling

$$
\frac{i}{\beta g_{s}} P\left[\left(\mathrm{i}_{X} \mathrm{i}_{X}\right) \mathrm{i}_{l} C^{(4)}\right]
$$

gives rise to a stable non-Abelian solution of the form

$$
F_{0 z i j k}^{(5)}=f \epsilon_{i j k}, \quad X^{i}=-\frac{1}{4} g_{s} f J^{i},
$$

which we interpret as $N$ Type IIB gravitational waves expanding into a D3-brane transverse to the propagation direction $X^{9}$ of the waves, and wrapped around the $Z$ direction. The rest of dielectric couplings in the action (4.5) describe multipole couplings to KK-monopoles and exotic branes.

These gravitational wave configurations represent a microscopical description of the dual giant gravitons of [14, 15], where we have however ignored the back reaction of the geometry.[2]

If we take into account the non-Abelian character of the actions for multiple fundamental strings and multiple waves that we have presented here and in our previous paper [9], it is remarkable how well the duality relations hold, both for the actions as for their solutions. Dielectric solutions describing fundamental strings expanding into D3 and D4-branes have been given in [9]. These solutions are T-dual to the solutions that we have just discussed for dielectric gravitational waves, just as one may expect by naively applying the duality relations shown in Figure 2. Also, S-duality acting on the Type IIB solutions gives the expected answer, as can be seen in this Figure: the F1-D3 configuration is S-dual to the well known D1-D3 solution, and the gravitational wave expanding into a D3-brane is clearly S-duality invariant.

There exists as well another type of polarisation effect, referred to as the magnetic moment effect [13], where $p$-branes with a non-zero velocity polarise under the influence of an external magnetic field (as opposed to an electric field, for the dielectric effect). In this reference it was shown that a set of $N$ D0-branes in an external magnetic flux tube, with a non-zero velocity along the flux tube, can be described by a Lagrangian

$$
L=\operatorname{STr}\left\{\frac{1}{2} \dot{X}^{2}+\frac{1}{4 g_{s}^{2}}[X, X]^{2}+\frac{i}{6 g_{s}} \dot{X}^{l} X^{k} X^{j} X^{i} F_{i j k l}^{(4)}\right\}
$$

\footnotetext{
${ }^{11}$ Recall that the pull-backs are defined in terms of covariant derivatives 3.5.).

${ }^{12}$ Recall that one has to go beyond the linear approximation presented in this paper in order to describe properly giant gravitons in an $A d S_{m} \times S^{n}$ space.
} 


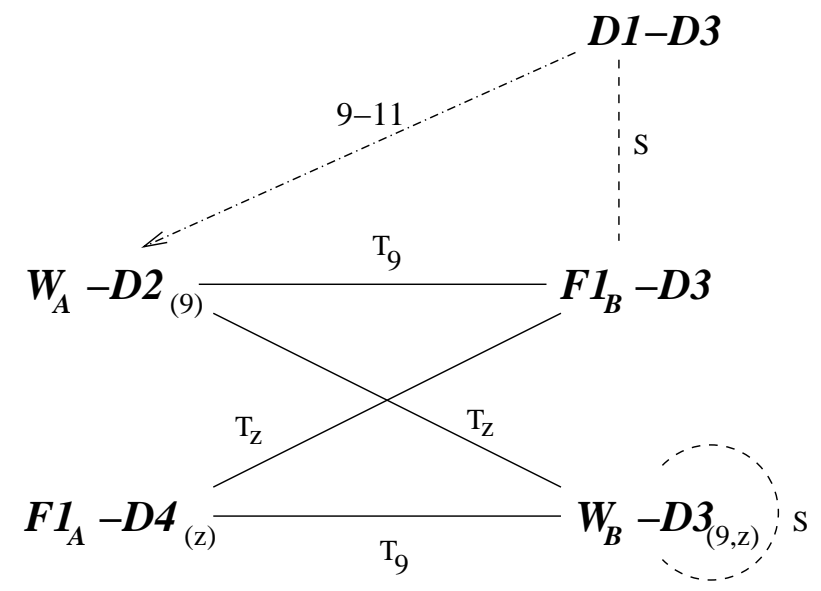

Figure 2: Duality relations between solutions corresponding to dielectric fundamental strings and gravitational waves in the Type IIA and Type IIB theories. In our notation $W_{A}-D 2$ represents multiple Type IIA waves expanded into a D2-brane. As in Figure 1. $T_{9}$ denotes a T-duality transformation in the propagation direction $X^{9}$ of the waves, or world sheet spatial direction of the strings, while $T_{z}$ indicates a T-duality transformation in a direction orthogonal to the waves and the strings. Also shown is the S-duality between the D1-D3 solution and the F1-D3 solution in Type IIB, the S-duality invariance of Type IIB gravitational waves expanding into a D3-brane and the 9-11 flip that maps the D1-D3 solution into expanding Type IIA gravitational waves. The lower indices between brackets indicate the isometry directions of the corresponding world volume theory.

where $\dot{X}$ indicates differentiation with respect to the world line coordinate $\tau$. This Lagrangian admits a solution of the form

$$
F_{i j k l}^{(4)}=f \epsilon_{i j k l}, \quad X^{a}=\frac{1}{4} g_{s} f v J^{a}, \quad X^{4}=v \tau \mathbb{1},
$$

where the coordinates $i$ are split as $i=(a, 4), X^{4}$ is the direction on which the D0-branes move, and, as in (5.3), the matrices $J^{a}$ form an $N \times N$ representation of $S U(2)$. This solution describes D0-branes expanding into a D2-brane orthogonal to the direction $X^{4}$ in which the D0-branes move. The radius of the D2-brane turns out to be independent of $N$, but proportional to the momentum of the D0-branes, at least for $N \gg 1$. This solution was compared to a spherical D2-brane (with the 2-sphere in the $(a, b, c)$-directions) embedded in a magnetic R-R flux tube and moving in the $X^{4}$ direction, and it was found that both the radius and the energy of the D2-brane coincide with those provided by the microscopical picture, in terms of expanding D0-branes. As also discussed in [13], the eleven-dimensional interpretation of (5.7) should be in terms of gravitons, moving both along the eleventh direction and along $X^{4}$, polarising into a spherical M2-brane. Therefore, this solution provides a microscopical description of a particular eleven-dimensional giant graviton configuration.

Following the construction of [13] it is not difficult to see that the dielectric coupling (5.1) for multiple Type IIA gravitational waves gives rise to the same Lagrangian (5.6), now with gauge covariant pull-backs into the world line. A solution identical to (5.7) will therefore also describe ten-dimensional gravitational waves moving in the $X^{9}$ and in the $X^{4}$ directions, expanding into a D2-brane transverse to the $X^{9}$ direction. This is just the dimensionally reduced version of the eleven-dimensional picture of [13], as we will further discuss in the conclusions.

Reference [13] considered as well a solution corresponding to eleven-dimensional gravitons 
propagating only in the $X^{9}$ direction. The corresponding Lagrangian is similar to (5.6), but with a magnetic field strength with $F_{i j k 9}^{(4)}$ components. Reduction over $X^{9}$ yields a system of D0-branes in a magnetic NS-NS field $H_{i j k}$, where the coupling arises from the Born-Infeld, rather than from the Chern-Simons action (see [2]). It would be interesting to construct the analogous version of this solution in terms of Type IIA gravitational waves in a $F_{i j k 9}^{(4)}$ flux tube. However, due to the fact that in our approach the propagation direction $X^{9}$ of the waves is isometric, it is not clear how to obtain from the action (3.1) a coupling of the form

$$
\mathrm{S} \operatorname{Tr}\left\{\dot{X}^{9} X^{k} X^{j} X^{i} F_{i j k 9}^{(4)}\right\},
$$

a difficulty that should be related to the problem, that we have already discussed, of restoring the dependence in the isometric direction in the non-Abelian case.

\section{Conclusions}

We have provided some evidence that Matrix string theory describes, in the static gauge, coincident Type IIA gravitational waves. We have given some weight to this identification by showing that Matrix string theory in static gauge and in a weakly coupled background reproduces in the $g_{s} \rightarrow 0$ limit, the linearised action describing Abelian Type IIA gravitational waves in a weakly coupled background, and by showing agreement with previous results derived from string dualities [19].

The action thus proposed contains the linear couplings of non-Abelian gravitons to closed string fields. These terms include non-Abelian, Myers-like couplings that are responsible for the expansion of the gravitons into higher-dimensional branes. We have studied some particular configurations, corresponding to multiple gravitational waves expanding into a spherical D2-brane, in the presence of an external electric flux tube, and in a spherical D2brane with non-zero transverse velocity, in the presence of an external magnetic flux tube. We related these solutions to similar configurations in eleven dimensions [13].

Perhaps the most interesting dielectric effect associated to gravitational waves is the one giving rise to the giant graviton configurations in $A d S_{m} \times S^{n}$ backgrounds. We would need however to go beyond the linear order approximation of this paper in order to provide a microscopical description of these configurations, given that a linear perturbation of a flat background is not an adequate framework in which to describe the $A d S$ background. It should however be possible to study giant graviton configurations in the Penrose limit, given that the pp-wave background can be approximated by a linear perturbation to Minkowski[3, or in more general backgrounds, like those presented in [13, 16, 17].

We have also provided a Matrix theory description for non-Abelian gravitons in the Type IIB theory. The key ingredient is the T-duality connection between Type II waves, that we have applied to the Matrix string theory action in static gauge in a weakly coupled background. The action thus obtained reproduces in the $g_{s} \rightarrow 0$ limit a particular linearised action describing Abelian Type IIB gravitational waves. A non-trivial check of this action is its S-duality invariance, a property that had been predicted by the analysis of the Type IIB spacetime supersymmetry algebra [35]. We have also given a non-Abelian solution of Type IIB gravitons expanding into a dielectric D3-brane.

\footnotetext{
${ }^{13} \mathrm{An}$ analysis of eleven-dimensional gravitational waves with dielectric couplings in a pp-wave background, arising as the Penrose limit of $A d S_{4} \times S^{7}$ and $A d S_{7} \times S^{4}$, has been given in 18 .
} 
Finally, we should stress an important point about the actions for gravitational waves that we have derived. If we start with the Matrix theory action describing Type IIA waves and perform a T-duality transformation along the direction of propagation of the waves, we arrive at a Matrix theory formulation of Type IIB fundamental strings in the static gauge [9], to which we have already referred in this article. As shown in [9], this action is actually S-dual to the world volume effective action describing multiple D-strings, a fact that suggests that this Matrix description of Type IIB F-strings, and therefore the one here proposed to describe Type IIA waves, are perhaps only suitable to describe the corresponding branes in the strong coupling regime.

Moreover, the Matrix theory description of Type IIA waves is in terms of the non-Abelian extension of a particular description for a ten-dimensional massless particle, in terms of an action in which the direction of propagation of the particle plays a special role (it is isometric). This action is related to the usual description of the ten-dimensional massless particle by means of a Legendre ( $\leftrightarrow$ world volume duality) transformation. Now, world volume duality transformations typically map the weak and the strong coupling regimes of a theory. Therefore, it is possible that the dielectric configurations that we have studied in section 5 are suitably described by the Myers-like couplings that we have computed in this paper only in the strong coupling regime. Still, dielectric configurations of this type are expected to occur as well in the weak coupling regime, at least those preserving some fraction of the supersymmetries, though perhaps they are described microscopically in terms of different variables. It would be quite interesting to further study whether this is really the case.

One remarkable aspect of our description of non-Abelian Type IIA waves is that it can be used to describe non-Abelian gravitons in M-theory. Indeed, since it adequately describes Type IIA gravitons in the strong coupling regime, we can describe eleven-dimensional gravitons by just rewriting ten-dimensional fields in terms of eleven-dimensional ones. The coupling (5.1):

$$
\frac{i}{\beta g_{s}} P\left[\left(\mathrm{i}_{X} \mathrm{i}_{X}\right) C^{(3)}\right],
$$

that we used to construct the dielectric solution associated to Type IIA waves expanded into a D2-brane, does actually agree with the dipole coupling for eleven-dimensional waves predicted in [19]. This solution is in fact the dimensional reduction of $N$ eleven-dimensional gravitational waves polarised into a spherical M2-brane, with the 2 -sphere in the $(i, j, k)$-space, where the reduction has to be performed in a direction orthogonal to both the direction of propagation and $X^{i}, X^{j}$ and $X^{k}$. N M-theory gravitons expanded in a spherical M2-brane have been considered in [18], in the pp-wave background. The off-diagonal terms in the ppwave metric give a mass to the embedding scalars in the non-Abelian action, and the solution can be made supersymmetric by an appropriate choice of the mass. Dielectric solutions with non-zero masses were considered first in 断. On the other hand, a reduction of the elevendimensional solution along the propagation direction $X^{9}$ of the waves yields the well-known solution associated to $N$ D0-branes expanding into a dielectric D2-brane, already studied by Myers in [2]. In fact, a nice check of the eleven-dimensional action for the M-theory gravitons would be to reproduce the linearised action for multiple D0-branes [3] when reducing along the direction of propagation of the gravitons.

It would also prove interesting to derive the action for M-theory gravitons directly in a Matrix theory set-up, i.e. using Matrix theory in a weakly coupled background [36, 37]. A Matrix theory description of M-theory gravitons in a pp-wave background has recently been 
given in 18, 38, 39 [4]. This Matrix model is determined by the supersymmetry algebra of the pp-wave background, and consists on the usual Matrix theory of BFSS 441 plus a correcting term with a dielectric coupling which is shown to be responsible for the expansion of the gravitons into a fuzzy M2-brane. It would be interesting to try to make contact with these results within our description. We hope to report progress in this direction in a forthcoming publication [42].

\section{Acknowledgements}

We wish to thank Dominic Brecher, Roberto Emparan, Clifford Johnson, Nakwoo Kim, Tomás Ortín, M.A.R. Osorio and Dave Page for the useful discussions. B.J. thanks the Instituto de Física Teórica of the U.A.M./C.S.I.C. in Madrid and the Departamento de Física of the Universidad de Oviedo for their hospitality during part of this work. Y.L. would also like to thank the Institute for Theoretical Physics in Leuven and the AEI in Potsdam for hospitality.

\section{A Matrix currents}

In this Appendix we include, for completeness, the explicit expressions for the currents that couple in the gravitational wave actions that we have constructed. Here we have taken static gauge (3.3) as compared to [3]. This results in the fact that all terms proportional to $D_{\sigma} X$ reduce to terms proportional to $[A, X]$. Note also that our currents differ from the ones in [3], due to the fact that we use dimensionless world sheet coordinates

$$
\sigma=\frac{g_{s}}{\sqrt{\alpha^{\prime}}} x, \quad \tau=\frac{R}{\alpha^{\prime}} t
$$

and have performed the rescalings $X^{i} \longrightarrow \sqrt{\alpha^{\prime}} X^{i}$ and $g_{s} \longrightarrow g_{s} /(2 \pi)$. This results in practise in a rescaling of the currents, with respect to [3], by a factor of $\beta=R / \sqrt{\alpha^{\prime}}$ for each term of the form $\dot{X},[X, X],[A, X]$ and $\dot{A}$, and a factor of $g_{s}$ and $1 / g_{s}$ for each $\dot{A}$ and $[X, X]$ respectively.

\footnotetext{
${ }^{14}$ See [40] for a formulation of Matrix string theory in a pp-wave background.
} 


\section{A.1 R-R currents}

With $i, j=1, \ldots, 8$, and up to order $\beta^{2}$, we have, for the Type IIA waves:

$$
\begin{aligned}
I_{0}^{0} & =\mathbb{1} \\
I_{0}^{i} & =\beta \dot{X}^{i}, \\
I_{0}^{9} & =g_{s} \beta \dot{A}, \\
I_{2}^{0 i j} & =-\frac{i \beta}{6 g_{s}}\left[X^{i}, X^{j}\right] \\
I_{2}^{09 i} & =-\frac{i \beta}{6}\left[A, X^{i}\right] \\
I_{2}^{i j k} & =-\frac{i \beta^{2}}{6 g_{s}}\left(\dot{X}^{i}\left[X^{j}, X^{k}\right]+\dot{X}^{j}\left[X^{k}, X^{i}\right]+\dot{X}^{k}\left[X^{i}, X^{j}\right]\right), \\
I_{2}^{9 i j} & =-\frac{i \beta^{2}}{6}\left(\dot{A}\left[X^{i}, X^{j}\right]-\dot{X}^{i}\left[A, X^{j}\right]+\dot{X}^{j}\left[A, X^{i}\right]\right), \\
I_{4}^{0 i j k l} & =-\frac{\beta^{2}}{2 g_{s}^{2}}\left(\left[X^{i}, X^{j}\right]\left[X^{k}, X^{l}\right]+\left[X^{i}, X^{k}\right]\left[X^{l}, X^{j}\right]+\left[X^{i}, X^{l}\right]\left[X^{j}, X^{k}\right]\right), \\
I_{4}^{09 i j k} & =-\frac{\beta^{2}}{2 g_{s}}\left(\left[A, X^{i}\right]\left[X^{j}, X^{k}\right]+\left[A, X^{j}\right]\left[X^{k}, X^{i}\right]+\left[A, X^{k}\right]\left[X^{i}, X^{j}\right]\right), \\
I_{4}^{i j k l m} & =-\frac{15 \beta^{3}}{2 g_{s}^{2}} \dot{X}^{[i}\left[X^{j}, X^{k}\right]\left[X^{l}, X^{m]}\right], \\
I_{4}^{9 i j k l} & =-\frac{3 \beta^{3}}{2 g_{s}}\left(\dot{A}\left[X^{[i}, X^{j}\right]\left[X^{k}, X^{l]}\right]-4 \dot{X}^{[i}\left[X^{j}, X^{k}\right]\left[A, X^{l]}\right]\right), \\
I_{6}^{0 i j k l m n} & =\frac{i \beta^{3}}{g_{s}^{3}}\left[X^{[i}, X^{j}\right]\left[X^{k}, X^{l}\right]\left[X^{m}, X^{n]}\right], \\
I_{6}^{09 i j k l m} & =\frac{i \beta^{3}}{g_{s}^{2}}\left[A, X^{[i}\right]\left[X^{j}, X^{k}\right]\left[X^{l}, X^{m]}\right], \\
I_{6}^{i j k l m n p} & =\frac{7 \beta^{4}}{g_{s}^{3}} \dot{X}^{[i}\left[X^{j}, X^{k}\right]\left[X^{l}, X^{m}\right]\left[X^{n}, X^{p]}\right], \\
I_{6}^{9 i j k l m n} & =\frac{i \beta^{4}}{g_{s}^{2}}\left(\dot{A}\left[X^{[i}, X^{j}\right]\left[X^{k}, X^{l}\right]\left[X^{m}, X^{n]}\right]-6 \dot{X}^{[i}\left[X^{j}, X^{k}\right]\left[X^{l}, X^{m}\right]\left[A, X^{n]}\right]\right) .
\end{aligned}
$$

To obtain the currents that couple in the linear action (4.2) for Type IIB gravitational waves, one of the transverse coordinates, say $X^{8}$, must be taken to be $Z \equiv g_{s} \omega$.

\section{A.2 NS-NS currents}

The NS-NS currents that couple in the action for Type IIA gravitational waves can simply be obtained from the Matrix string currents in [9] going to static gauge. The corresponding NS-NS currents for Type IIB gravitational waves have however singled out the $Z$ direction, and we will include their explicit expressions for the sake of clarity. They are given, up to 
$\operatorname{order} \beta^{2}$, by:

$$
\begin{aligned}
I_{\phi} & =\mathbb{1}-\frac{\beta^{2}}{2} \dot{X}^{2}-\frac{\beta^{2}}{4 g_{s}^{2}}[X, X]^{2}-\frac{g_{s}^{2} \beta^{2}}{2}\left(F^{2}+\tilde{F}^{2}\right)-\frac{\beta^{2}}{2}\left([A, X]^{2}+[\omega, X]^{2}\right)-\frac{g_{s}^{2} \beta^{2}}{2}[A, \omega]^{2} \\
I_{h}^{00} & =\mathbb{1}+\frac{\beta^{2}}{2} \dot{X}^{2}-\frac{\beta^{2}}{4 g_{s}^{2}}[X, X]^{2}+\frac{g_{s}^{2} \beta^{2}}{2}\left(F^{2}+\tilde{F}^{2}\right)-\frac{\beta^{2}}{2}\left([A, X]^{2}+[\omega, X]^{2}\right)-\frac{g_{s}^{2} \beta^{2}}{2}[A, \omega]^{2}, \\
I_{h}^{0 i} & =\beta \dot{X}^{i} \\
I_{h}^{0 z} & =g_{s} \beta \tilde{F} \\
I_{h}^{09} & =g_{s} \beta F \\
I_{h}^{i j} & =\beta^{2} \dot{X}^{i} \dot{X}^{j}-\frac{\beta^{2}}{g_{s}^{2}}\left[X^{i}, X^{k}\right]\left[X^{k}, X^{j}\right]+\beta^{2}\left[A, X^{i}\right]\left[A, X^{j}\right]+\beta^{2}\left[\omega, X^{i}\right]\left[\omega, X^{j}\right], \\
I_{h}^{i z} & =g_{s} \beta^{2} \dot{X}^{i} \tilde{F}+\frac{\beta^{2}}{g_{s}}\left[X^{i}, X^{j}\right]\left[\omega, X^{j}\right]+g_{s} \beta^{2}\left[A, X^{i}\right][A, \omega], \\
I_{h}^{i 9} & =g_{s} \beta^{2} \dot{X}^{i} F+\frac{\beta^{2}}{g_{s}}\left[X^{i}, X^{j}\right]\left[A, X^{j}\right]-g_{s} \beta^{2}\left[\omega, X^{i}\right][A, \omega], \\
I_{h}^{z z} & =g_{s}^{2} \beta^{2} \tilde{F}^{2}+\beta^{2}[\omega, X]^{2}+g_{s}^{2} \beta^{2}[A, \omega]^{2}, \\
I_{h}^{99} & =g_{s}^{2} \beta^{2} F^{2}+\beta^{2}[A, X]^{2}+g_{s}^{2} \beta^{2}[A, \omega]^{2}, \\
I_{h}^{z 9} & =g_{s}^{2} \beta^{2} F \tilde{F}+\beta^{2}\left[\omega, X^{j}\right]\left[A, X^{j}\right], \\
I_{s}^{0 i} & =-\frac{i \beta^{2}}{2 g_{s}}\left[X^{i}, X^{j}\right] \dot{X}^{j}+\frac{i g_{s} \beta^{2}}{2}\left[A, X^{i}\right] F+\frac{i g_{s} \beta^{2}}{2}\left[\omega, X^{i}\right] \tilde{F}, \\
I_{s}^{0 z} & =-\frac{i \beta^{2}}{2}\left[\omega, X^{j}\right] \dot{X}^{j}+\frac{i g_{s}^{2} \beta^{2}}{2} F[A, \omega], \\
I_{s}^{09} & =-\frac{i \beta^{2}}{2} \dot{X}^{i}\left[A, X^{i}\right]-\frac{i g_{s}^{2} \beta^{2}}{2} \tilde{F}[A, \omega], \\
I_{s}^{i j} & =\frac{i \beta}{2 g_{s}}\left[X^{i}, X^{j}\right], \\
I_{s}^{z i} & =\frac{i \beta}{2}\left[\omega, X^{i}\right] \\
I_{s}^{9 i} & =\frac{i \beta}{2}\left[A, X^{i}\right] \\
I_{s}^{9 z} & =\frac{i g_{s} \beta}{2}[A, \omega], \\
&
\end{aligned}
$$

where $Z=g_{s} \omega, F=\partial A, \tilde{F}=\partial \omega$, and

$$
\begin{aligned}
{[X, X]^{2} } & \equiv\left[X^{i}, X^{j}\right]\left[X^{i}, X^{j}\right] \\
{[A, X]^{2} } & \equiv\left[A, X^{i}\right]\left[A, X^{i}\right] \\
{[\omega, X]^{2} } & \equiv\left[\omega, X^{i}\right]\left[\omega, X^{i}\right] .
\end{aligned}
$$

It is straightforward to check that $I_{\phi}, I_{h}^{a b}, I_{s}^{a b}$ and $I_{s}^{9 z}$ are singlets under the S-duality transformations (4.13), while $\left(I_{h}^{a 9}, I_{h}^{a z}\right)$ and $\left(I_{s}^{a 9}, I_{s}^{a z}\right)$ transform as doublets. $I_{h}^{z 9}$ changes sign, and $I_{h}^{99}$ and $I_{h}^{z z}$ get mapped into each other. As shown in subsection 4.2, these currents combine in the linear action (4.2) with the background fields in such a way that the action is invariant under S-duality transformations. 


\section{B Gravitational background fields and duality}

In this Appendix we summarise the duality properties of the gravitational fields that arise in the different duality transformations that have been carried out in the paper, and briefly summarise previous results in the literature concerning the role they play as sources for gravitational branes in Type II. We refer the reader to [33] for a more extensive study.

Tables 1 and 2 summarise the Type II gravitational branes that are charged w.r.t. these fields. Some of these branes are exotic branes, in the sense that they are not predicted by the standard Type II spacetime supersymmetry algebras (see however [32]). Exotic branes have been encountered in the literature in different contexts. On the one hand their existence is required in order to fill up multiplets of BPS states in representations of the U-duality groups $E_{7}(\mathbb{Z}), E_{8}(\mathbb{Z})$ of M-theory on $T^{7}, T^{8}$ tori $[43$, 29, 30, 31, 44]. On the other hand they are predicted by dualities on branes that do occur as central charges in the spacetime supersymmetry algebras 33 .

We have summarised in Figure 3 the duality relations between the different exotic branes, as well as between exotic and ordinary origins. The duality relations among the background fields that couple minimally to these branes are also indicated in this figure. These connections are of particular relevance for the calculations that we have carried out in the paper.

\begin{tabular}{|c|c|}
\hline Background field & Brane \\
\hline \hline$\tilde{B}$ & NS5 \\
\hline$i_{k} N^{(7)}$ & KK-monopole \\
\hline$i_{k} N^{(8)}$ & $\left(6,1^{2} ; 3\right)$ \\
\hline$i_{k} N^{(9)}$ & $\left(7,1^{3} ; 3\right)$ \\
\hline
\end{tabular}

Table 1: Higher form NS-NS and gravitational background fields of the Type IIA superstring

In Table 1, we summarise the higher form NS-NS and gravitational background fields of the Type IIA superstring, and the branes, some of them exotic, to which they couple minimally. Our notation for the exotic branes is that of [30]. The first entry gives the number of ordinary spatial world volume directions, the entries of the form $m^{n}$ indicate that there are $m$ spatial directions which are gauged in the effective action and whose radii have $n$th power. Finally the last entry gives the power of the inverse of the string coupling constant. In this notation the KK-monopole is, for instance, denoted as $\left(5,1^{2} ; 2\right) . N^{(7)}$ couples in the world volume of the KK-monopole contracted with the Killing vector $k^{\mu}$, associated to its, isometric, TaubNUT direction. Similarly, $N^{(8)}$ and $N^{(9)}$ couple to exotic branes, contracted with the Killing vector associated to the isometry of the brane. $N^{(7)}$ is Poincaré dual to the Killing vector considered as a 1-form, $k_{\mu}$ [45], $N^{(8)}$ to its modulus and $N^{(9)}$ to the mass of the massive Type IIA supergravity that is obtained by reducing the massive eleven-dimensional supergravity of 22] along a direction different from the Killing direction [33].

In Table 2 we summarise some of the higher form NS-NS and gravitational background fields of the Type IIB superstring and the branes, some of them exotic, to which they couple minimally. The $\left(5,2^{2} ; 3\right)$-brane is related by T-duality to the Type IIA $\left(6,1^{2} ; 3\right)$-brane, with the T-duality taking place along a world volume direction of the IIA brane, direction which

\footnotetext{
${ }^{15}$ Predicted by the SUSY algebras.
} 


\begin{tabular}{|c|c|}
\hline Background field & Brane \\
\hline \hline$\tilde{B}$ & NS5 \\
\hline$i_{k} N^{(7)}$ & KK-monopole \\
\hline$i_{k} i_{l} N^{(8)}$ & $\left(5,2^{2} ; 3\right)$ \\
\hline$i_{k} i_{l} \mathcal{N}^{(8)}$ & $\left(5,2^{2} ; 2\right)$ \\
\hline$i_{k} i_{l} N^{(9)}$ & $\left(6,1^{2}, 1^{3} ; 3\right)$ \\
\hline
\end{tabular}

Table 2: Higher form NS-NS and gravitational background fields of the Type IIB superstring.

becomes isometric in the dual brane. The Type IIB $\left(5,2^{2} ; 2\right)$-brane on the other hand is related by T-duality to the Type IIA Kaluza-Klein monopole, with T-duality taking place along a transverse direction to the monopole. This direction becomes also isometric in the dual brane. Finally, the Type IIB $\left(6,1^{2}, 1^{3} ; 3\right)$-brane is related via T-duality along a transverse direction to the $\left(7,1^{3} ; 3\right)$-brane of Type IIA, with the duality direction becoming again isometric. In all these cases the $N^{(8)}, \mathcal{N}^{(8)}$ and $N^{(9)}$ fields couple to the world volume of the branes contracted with the two Killing vectors $k^{\mu}$ and $l^{\mu}$, associated to the two isometries of the brane. $N^{(8)}$ and $\mathcal{N}^{(8)}$ are Poincaré duals to the two scalars $k^{2}, l^{2}$. The field $N^{(9)}$ is dual to a mass parameter, which should be the one of the nine-dimensional Type II massive supergravity with a Killing isometry to which this 7-brane gives mass (see [33]). 


\section{References}

[1] R. Emparan, Phys. Lett. B423 (1998) 71, hep-th/9711106.

[2] R.C. Myers, JHEP 9912 (1999) 022, hep-th/9910053.

[3] W. Taylor, M. Van Raamsdonk, Nucl.Phys. B558 (1999) 63, hep-th/9904095.

[4] S.P. Trivedi, S. Vaidya, JHEP 0009 (2000) 041, hep-th/0007011.

[5] M. Costa, C. Herdeiro, L. Cornalba, Nucl. Phys. B619 (2001) 155, hep-th/0105023.

[6] R. Emparan, Nucl. Phys. B610 (2001) 169, hep-th/0105062.

[7] D. Brecher, P. Saffin, Nucl. Phys. B613 (2001) 218, hep-th/0106206.

[8] R. Schiappa, Nucl. Phys. B608 (2001) 3, hep-th/0005145.

[9] D. Brecher, B. Janssen, Y. Lozano, Nucl. Phys. B634 (2002) 23, hep-th/0112180.

[10] P.J. Silva, JHEP 0202 (2002) 004, hep-th/0111121.

[11] R. Dijkgraaf, E. Verlinde, H. Verlinde, Nucl. Phys. B500 (1997) 43, hep-th/9703030.

[12] J. McGreevy, L. Susskind, N. Toumbas, JHEP 0006 (2000) 008, hep-th/0003075.

[13] S.R. Das, S.P. Trivedi, S. Vaidya, JHEP 0010 (2000) 037, hep-th/0008203.

[14] M.T. Grisaru, R.C. Myers, Ø. Tafjord, JHEP 0008 (2000) 040, hep-th/0008015.

[15] A. Hashimoto, S. Hirano, N. Itzhaki, JHEP 0008 (2000) 051, hep-th/0008016.

[16] J.M. Camino, A.V. Ramallo, JHEP 0109 (2001) 012, hep-th/0107142.

[17] J.M. Camino, A.V. Ramallo, Phys. Lett. B525 (2002) 337, hep-th/0110096.

[18] D. Berenstein, J. Maldacena, H. Nastase, JHEP 0204 (2002) 013, hep-th/0202021.

[19] Y. Lozano, Phys. Rev. D64 (2001) 106011, hep-th/0012137.

[20] E. Bergshoeff, I. Entrop, R. Kallosh, Phys. Rev. D49 (1994) 6663, hep-th/9401025.

[21] E. Bergshoeff, B. Janssen, T. Ortín, Phys. Lett. B410 (1997) 131, hep-th/9706117.

[22] E. Bergshoeff, Y. Lozano, T. Ortín, Nucl. Phys. B518 (1998) 363, hep-th/9712115.

[23] E. Bergshoeff, J.P. van der Schaar, Class. Quant. Grav. 16 (1999) 23, hep-th/9806069.

[24] E. Eyras, B. Janssen, Y. Lozano, Nucl. Phys. B531 (1998) 275, hep-th/9806169.

[25] E. Bergshoeff, C.M. Hull, T. Ortín, Nucl.Phys. B451 (1995) 547, hep-th/9504081.

[26] W. Taylor, M. Van Raamsdonk, Nucl. Phys. B573 (2000) 703, hep-th/9910052.

[27] A. Dasgupta, H. Nicolai, J. Plefka, JHEP 0005 (2000) 007, hep-th/0003280.

[28] J. Plefka, Int. J. Mod. Phys. A16 (2001) 660, hep-th/0009193. 
[29] M. Blau, M. O’Loughlin, Nucl. Phys. B525 (1998) 182, hep-th/9712047.

[30] C.M. Hull, JHEP 9807 (1998) 018, hep-th/9712075.

[31] N.A. Obers, B. Pioline, E. Rabinovici, Nucl. Phys. B525 (1998) 163, hep-th/9712084.

[32] E. Lozano-Tellechea, T. Ortín, Nucl. Phys. B607 (2001) 213, hep-th/0012051.

[33] E. Eyras, Y. Lozano, Nucl. Phys. B573 (2000) 735, hep-th/9908094.

[34] See for example: J. Polchinski, String theory, Vol. 2: Superstring theory and beyond, Cambridge University Press (1998).

[35] C.M. Hull, Nucl. Phys. B509 (1998) 216, hep-th/9705162.

[36] D. Kabat, W. Taylor, Phys. Lett. B426 (1998) 297, hep-th/9712185.

[37] W. Taylor, M. van Raamsdonk, JHEP 9904 (1999) 013, hep-th/9812239.

[38] M. Cvetic, H. Lu, C.N. Pope, M-theory, pp-waves, Penrose limits and supernumerary supersymmetries, hep-th/0203229.

[39] K. Dasgupta, M. Sheikh-Jabbari, M. van Raamsdonk, JHEP 0205 (2002) 056, hepth/0205185.

[40] G. Bonelli, Matrix strings in pp-wave backgrounds from deformed super Yang-Mills theory, hep-th/0205213.

[41] T. Banks, W. Fischler, S. Shenker, L. Susskind, Phys. Rev. D55 (1997) 5112, hepth/9610043.

[42] B. Janssen, Y. Lozano, in preparation.

[43] S. Elitzur, A. Giveon, D. Kutasov, E. Rabinovici, Nucl. Phys. B509 (1998) 122, hepth/9707217.

[44] N.A. Obers, B. Pioline, Phys. Rept. 318 (1999) 113, hep-th/9809039.

[45] E. Bergshoeff, E. Eyras and Y. Lozano, Phys. Lett. B430 (1998) 77, hep-th/9802199. 

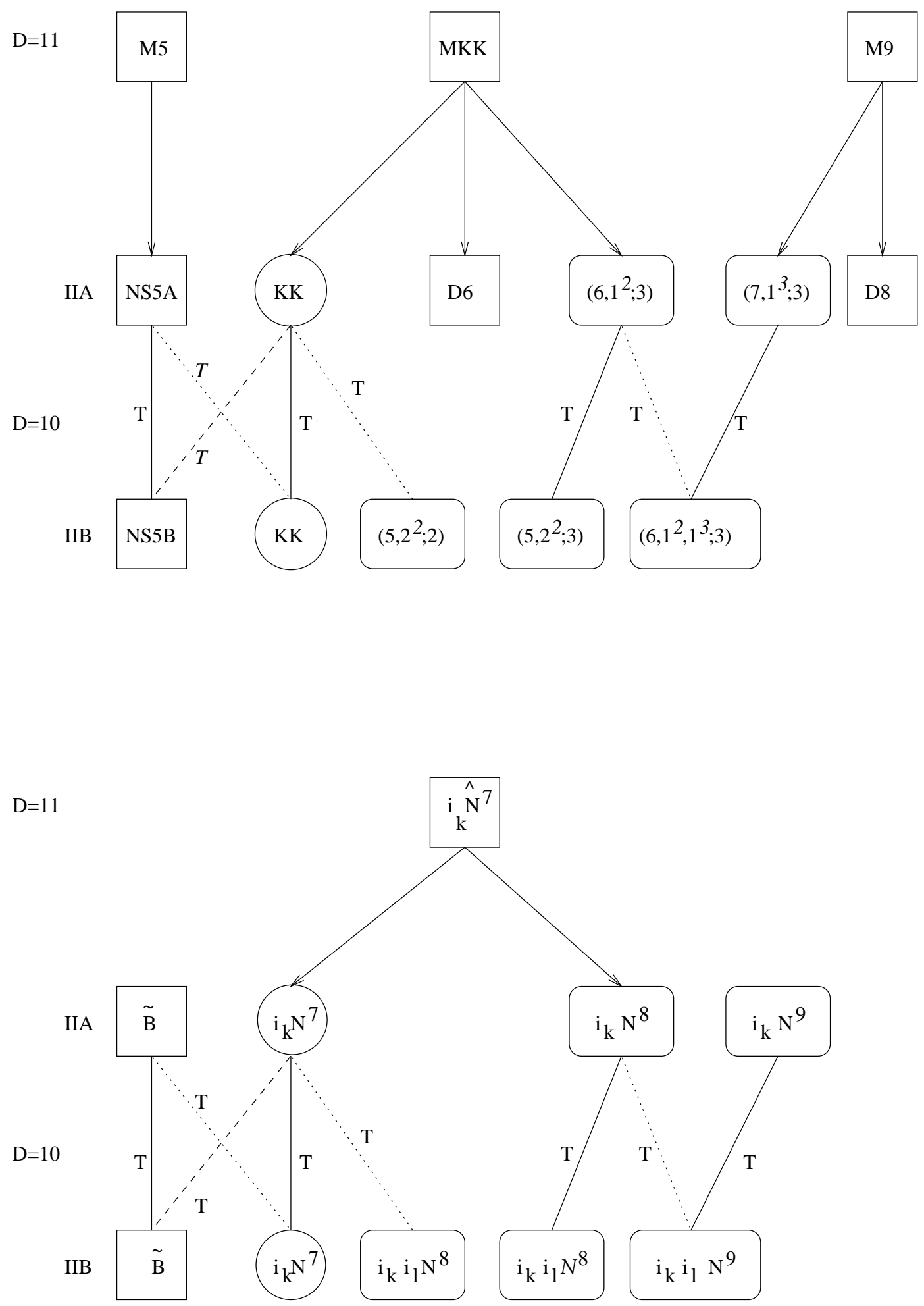

Figure 3: In these figures we show the relations under T-duality between the different gravitational branes and fields that we have encountered. We also include the M-theory origin of the Type IIA branes. With respect to the Type IIA branes, we indicate a T-duality along a world volume direction by a solid line, a T-duality along a Killing direction with a dashed line, and a T-duality along a transverse direction with a dotted line. 